\title{
Die Rolle des Internets für Gesundheitsinformationen in der Hals-Nasen-Ohrenheilkunde
}

\section{The role of the internet for healthcare information on ENT specific}

\section{issues}

아 (1) (요 $\ominus$

Autoren

Friedrich Ihler, Martin Canis

Institut

Klinik und Poliklinik für Hals-, Nasen-, Ohrenheilkunde in Kooperation mit dem Deutschen Schwindel- und Gleichgewichtszentrum, Klinikum der Universität München

\section{Schlüsselwörter}

Internet, Patients, Physician-Patient Relations, Health literacy, Health education

Bibliografie

DOI https://doi.org/10.1055/a-0801-2585

Laryngo-Rhino-Otol 2019; 98: S290-S312

(c) Georg Thieme Verlag KG Stuttgart · New York

ISSN 0935-8943

Korrespondenz

Prof. Dr. med. Friedrich Ihler

Univ. HNO-Klinik, Großhadern

Marchioninistraße 15

D-81377 München

friedrich.ihler@med.uni-muenchen.de

\section{ZUSAMMENFASSUNG}

Durch die Verbreitung des Internets kam es in den letzten Jahrzehnten zu einer Revolution des menschlichen Informations- und Kommunikationsverhaltens. Informationen sind durch das Internet leicht zugänglich und partizipative Anwendungen ermöglichen neue Formen der Interaktion. Das Gesundheitswesen ist unmittelbar davon betroffen, weil es wesentlich durch Information und Kommunikation geprägt ist. Der vorliegende Beitrag soll diese Entwicklung und ihre Auswirkungen in der Hals-NasenOhrenheilkunde darstellen.

Die Nutzung des Internets für die Suche nach Gesundheitsinformationen nimmt seit Jahren kontinuierlich zu und hat inzwischen eine erhebliche Bedeutung erreicht. Im klinischen Kontext haben derzeit noch andere Informationsquellen einen höheren Stellenwert. Für eine von Laien durchgeführte gesundheitsbezogene Recherche wird hauptsächlich die Suchmaschine Google verwendet. Obwohl die Beurteilung der Vertrauenswürdigkeit der dort dargebotenen Informationen schwer ist, haben sich alternative Angebote, die auf valide Gesundheitsinformationen spezialisiert sind, bisher nicht durchgesetzt. Anekdotische oder falsche Informationen treten regelmäßig auf.
Zahlreiche Studien haben die Qualität von Gesundheitsinformationen auf Internetseiten untersucht. Das methodische Spektrum dafür reicht von formelgebundenen Lesbarkeitsanalysen über strukturierte Beurteilungsinstrumente bis hin zu digitalen Zertifikaten. Im Ergebnis zeigt sich, dass Gesundheitsinformationen auf Internetseiten für die Allgemeinbevölkerung häufig schwer zu verstehen sind. Auch nahezu alle sozialen Medien enthalten Gesundheitsinformationen und ihre Bedeutung steigt. Dennoch gibt es zu Eigenschaften und Effekt von Gesundheitsinformationen in sozialen Medien bisher kaum wissenschaftliche Erkenntnisse. Die Verfügbarkeit von Gesundheitsinformationen im Internet macht ein neues Verständnis von Gesundheitskompetenz (health literacy) erforderlich. Das Konzept der digitalen Gesundheitskompetenz (eHealth literacy) beinhaltet u. a. Lesefähigkeit, Medienkompetenz, EDV-Kenntnisse und naturwissenschaftliche Grundbildung. Die Verwirklichung dieser Fähigkeiten wird durch individuelle und soziale Faktoren wie Bildung, sozioökonomischer Status und Alter beeinflusst. Bisherige Untersuchungen zeigten bei einem großen Teil der Patienten eine niedrige Gesundheitskompetenz.

Die Verbreitung des Internets verändert auch das Arzt-PatientenVerhältnis. Gut informierte Patienten fordern eine stärkere Beteiligung an medizinischen Entscheidungen ein. Ärzte haben eine besondere Verantwortung bei der Beratung medizinischer Laien durch Gewichtung und Verifizierung von Informationen. Ärzte sollten durch aktive Beteiligung dazu beitragen, dass die Digitalisierung der Medizin insgesamt den Patienten zugutekommt. Medizinische Fachgesellschaften sind in besonderer Weise dazu aufgerufen, diesen Prozess mit zu gestalten.

\section{ABSTRACT}

The wide distribution and availability of the internet during the last decades revolutionized the human information and communication behavior. Via internet, information can be easily retrieved and participative applications allow new types of interaction. The healthcare system is directly affected because information and communication represent a relevant part of it. The present contribution is intended to describe this development and its impact on otorhinolaryngology. The use of the internet for the research of health-related information is continuously increasing since several years and has meanwhile achieved significant importance. In the clinical context, other infor- 
mation sources still have a higher relevance. Laypeople mostly use the search engine of Google when performing health-related research. Even if the reliability of the presented information is difficult to assess, alternative offers that are specialized on valid healthcare information could not prevail. Anecdotic or incorrect information are regularly observed.

Numerous trials investigated the quality of healthcare information on web pages. The methodical spectrum reaches from formula-depending readability testing via structured assessment tools up to certificates. The result shows that healthcare information on internet sites is often difficult to understand for the general population. Nearly all social media contain healthcare information and their relevance is increasing. Nonetheless, there is only few scientific knowledge on the characteristics and the effect of healthcare information in social media.
The availability of online healthcare information requires new understanding of health literacy. The concept of digital literacy (eHealth literacy) contains among others the readability, media competence, IT knowledge, and basic scientific knowledge. The implementation of those skills depends on individual and social factors such as education, socio-economic status, and age. Investigations revealed a low healthcare literacy in a high percentage of the patients.

The distribution of the internet also modifies the relationship between physician and patient. Well-informed patients request being involved in medical decisions. Physicians have a particular responsibility regarding the consultation of medical laypeople by weighting and verifying information. By actively participating, physicians should contribute to digitization in medicine for the benefit of their patients. Medical associations are particularly invited to contribute to this process.

\section{Inhaltsverzeichnis}

$\begin{array}{ll} & \text { Zusammenfassung } \\ \text { 1. } & \text { Mbstract } \\ 2 . & \text { Einleitung } \\ 3 . \quad \text { Das Internet als Zugangsweg zu Gesundheitsinformationen } \\ 3.1 \quad \text { Entwicklung der Nutzung des Internets für } \\ \text { Gesundheitsinformationen } \\ \text { 3.2 } \quad \text { Nechnische Aspekte bei Gesundheitsrecherchen durch Laien } \\ \text { 4. } \quad \text { Qualität von Gesundheitsinformationen im Internet }\end{array}$

\section{Methoden}

Für diese Übersichtsarbeit wurde eine Literaturrecherche über Pubmed in der Datenbank MEDLINE durchgeführt. Es wurde hierbei für die Suchanfrage die folgende Formulierung verwendet:

„Internet“[Mesh] AND „Patients“[Mesh] OR „Consumer Health Information“[Mesh] AND „Internet“[Mesh] OR „Physician-Patient Relations“[Mesh] AND „Internet“[Mesh] OR digital patient empowerment OR dr. google[ti] OR dr google[ti].

Die Suchanfrage wurde zuletzt am 02. Juli 2018 aktualisiert. Berücksichtigt wurden nur englisch- und deutschsprachige Artikel. Dies führte zu 3589 Treffern. Alle Suchergebnisse wurden nach Originalarbeiten und Übersichtsarbeiten evaluiert, welche Gesundheitsinformationen im Internet und deren Auswirkung auf die Gesundheitskompetenz sowie das Arzt-Patienten-Verhältnis entweder allgemein oder in der Hals-Nasen-Ohrenheilkunde untersuchen.

In einem mehrstufigen Auswahlprozess nach PRISMA (Preferred Reporting Items for Systematic Reviews and Meta-Analyses) [1,2] resultierten daraus 108 Publikationen, die in diese qualitative Übersichtsarbeit eingeschlossenen wurden. Zusätzliche Publikationen wurden manuell sowie durch Rückwärts- oder Kontextsuche identifiziert. Ein Schema des Literaturauswahlprozesses findet sich in $\mathbf{A} \mathbf{A b b} \mathbf{~} \mathbf{~}$.

$\begin{array}{lll}4.1 & \text { Methoden } & 295 \\ 4.2 & \text { Untersuchungen } & 296 \\ 4.3 & \text { soziale Medien } & 296 \\ 5 . & \text { digitale Gesundheitskompetenz } & 302 \\ 5.1 & \text { Kompetenz und Gesundheit } & 302 \\ 5.2 & \text { Faktoren } & 303 \\ 5.3 & \text { Verteilung } & 303 \\ 6 . & \text { Arzt-Patienten-Verhältnis } & 304 \\ 6.1 & \text { Wandel durch das Internet } & 304 \\ 6.2 & \text { Chancen für Verbesserung } & 304 \\ 7 . & \text { Schlussfolgerungen, Ausblick und weiterführende Ansätze } & 305 \\ & \text { Literatur } & 306\end{array}$

\section{Einleitung}

Das Internet ist eine Verbindung von Computern und Computernetzwerken, die dem Austausch von Informationen dient [3]. Da das Internet in etwa einem Vierteljahrhundert die Kommunikation in nahezu allen Aspekten des menschlichen Lebens verändert hat [4], wird seine Entwicklung schon jetzt als ein wichtiger kulturhistorischer Meilenstein der Menschheit betrachtet [5]. Ein grundlegendes Funktionsprinzip ist die Übermittlung von digitalen Daten als Paket (packet switching). Dies erlaubt eine dezentrale Verknüpfung verschiedener Netzwerke [3]. Der Datenaustausch wird durch Netzwerkprotokolle geregelt, deren wichtigste Familie als Transmission Control Protocol/Internet Protocol (TCP/IP) bezeichnet wird. Diese wurden im Jahr 1974 von Cerf und Kahn formuliert [6].

Das World Wide Web (WWW) ist ein Teilbereich des Internets, der optimiert ist um strukturierte digitale Dokumente abzurufen. Diese werden als Internetseiten bezeichnet und über geeignete Programme (Browser) dargestellt. Das World Wide Web umfasst inzwischen einen sehr ausgedehnten Wissensraum, welcher grundsätzlich allen Menschen offensteht. Internetseiten sind hierbei in einer maschinenlesbaren Auszeichnungssprache verfasst; die gebräuchlichste wird als Hypertext Markup Language (HTML) bezeichnet. Eine 


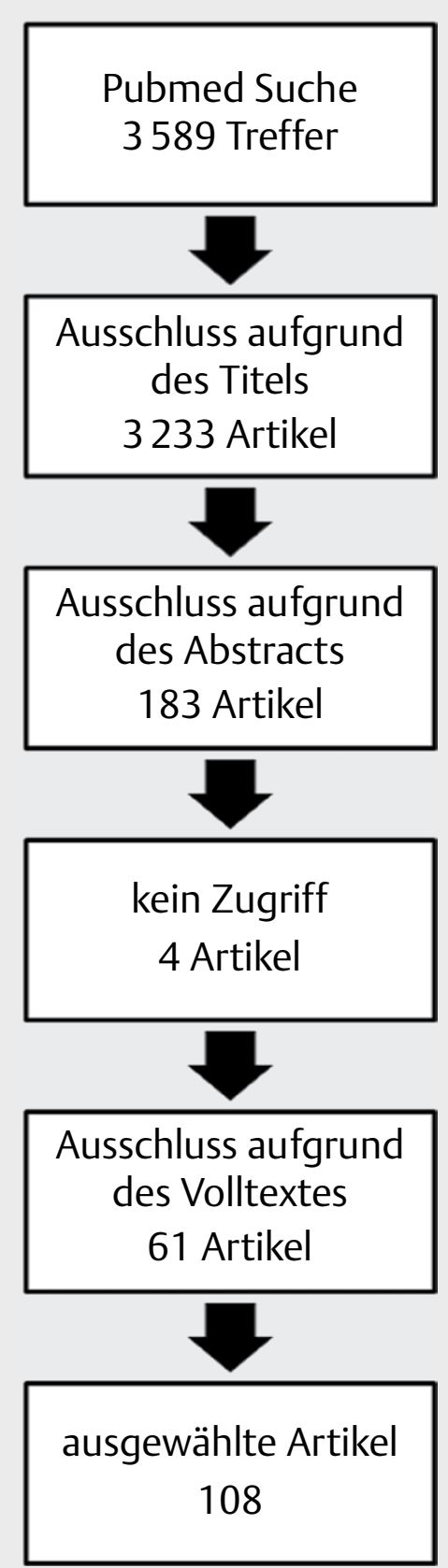

Abb. 1 Schema des Literaturauswahlprozesses.

Übertragung solcher Dokumente erfolgt über Protokolle aus der Hypertext Transfer Protocol-Familie (HTTP) [7]. Damit fungiert das Internet heute gleichzeitig als Rundfunkeinrichtung, als Verteiler von Informationen sowie als Medium für die Zusammenarbeit und Kommunikation über beliebige Distanzen hinweg $[3,4]$.

Suchmaschinen dienen der Erschließung der im Internet verfügbaren Inhalte und sind wahrscheinlich eine der wichtigsten Anwendung des Internets [8]. Sie erschließen automatisiert große Teile des Internets durch Indexierung [9]. Der Index einer Suchmaschine wird kontinuierlich aktualisiert und steht dadurch für Suchanfragen zur Verfügung. Als Antwort auf eine Suchanfrage gibt eine Suchmaschi- ne Ergebnisse aus, deren Rangfolge einer spezifischen Systematik folgt. Die bei weitem verbreitetste Suchmaschine ist Google. Grundlage des Erfolges von Google ist eine innovative Art, Suchergebnisse auf der Grundlage der darauf verweisenden Links nach der Bedeutung zu reihen [10].

Etwa seit der Jahrtausendwende hat sich das ursprüngliche Internet noch einmal grundlegend weiterentwickelt. Es stellt nun ein Medium dar, das gekennzeichnet ist durch einfache Bedienbarkeit (usability), Unabhängigkeit von Plattformen und Endgeräten (interoperability) sowie durch Inhalte, die von Nutzern erstellt werden (user-generated content). Beispiele hierfür sind neue Endgeräte wie Smartphones und soziale Medien. Diese Prinzipien werden zusammengefasst als Web 2.0, social web oder participatory web bezeichnet [11-13]. Wichtige Beispiele für soziale Medien sind Twitter, Wikipedia und Youtube.

Twitter existiert seit 2006 und ermöglicht den Austausch von Textnachrichten mit maximal 280 Zeichen sowie von Bildern und Videos. Neben der Funktion als soziales Medium hat Twitter auch Eigenschaften eines Massenmediums [14]. Twitter hat über 300000000 aktive Nutzer, die täglich mehrere hundert Millionen Nachrichten (Tweets) verfassen. Einzelne Tweets werden im Internet und den klassischen Medien breit rezipiert. Wikipedia ist eine Plattform, welche die kollaborative Erstellung einer Enzyklopädie durch Nutzer ermöglicht $[15,16]$. Seit dem Beginn im Jahr 2001 haben über 75000000 Nutzer mehr als 48000000 Artikel in über 300 Sprachen verfasst. Es wird als die umfassendste Enzyklopädie der Welt angesehen. Obwohl die Ersteller in ihrer Mehrzahl Laien sind und die Plattform in einer sehr flachen Hierarchie organisiert ist, erreicht Wikipedia eine erstaunliche Richtigkeit, wie in einem Vergleich mit der traditionsreichen englischsprachigen Enzyklopädie Encyclopædia Britannica dargestellt wurde [17]. Youtube ist eine seit 2005 aktive Plattform für den Austausch von Videos. Nutzer können dort Videos einstellen, ansehen, kommentieren und bewerten. Mit der Anzahl der Aufrufe entsteht zudem ein Maß für die Popularität eines Beitrags. Es ist noch nicht im Detail geklärt, welchen Gesetzmäßigkeiten die Popularität von Beiträgen folgt und wie es zum Phänomen der viralen Verbreitung kommt [18].

Komplexe Wechselwirkungen zwischen Erstellern, Nutzern und Inhalten zeichnen soziale Medien aus. Charakteristische Interaktionen in sozialen Medien wie „folgen“ (follow) und "gefallen“ (like) führen zu einer neuartigen Rückkopplung zwischen den Urhebern und Konsumenten von Inhalten. Dabei entsteht eine kollektive Meinung oder Bewertung, die genauer oder ausgewogener sein kann als eine individuelle Ansicht. Dies es Phänomen wird als „Weisheit der Vielen“ (wisdom of the crowd) bezeichnet und ist in sozialen Netzwerken bspw. für Produktbewertungen untersucht worden [19]. Die Motivationslage von Menschen, die in Internet-Gemeinschaften (online communities) Wissen einbringen, ist noch nicht vollständig geklärt. Wahrscheinlich ist es eine Mischung aus extrinsischer Motivation durch Wahrnehmung und intrinsischer Motivation durch Stärkung des Selbstwertgefühls [20].

Medien spielen in modernen Gesellschaften bei der Ausbildung des individuellen Verständnisses von Gesundheit und Krankheit und der damit verbundenen Werte eine komplexe und wesentliche Rolle $[21,22]$. Damit ist klar, warum eine Umwälzung der Medienlandschaft, wie durch die digitalen Medien geschehen, auch eine Veränderung im gesundheitsbezogenen Verhalten bewirkt. Die moderne Medizin ist ohne Informationstechnologie undenkbar geworden. 
Dementsprechend haben Änderungen in diesem Bereich eine profunde Auswirkung auf das Gesundheitswesen [23]. Das Internet ist dabei ein Medium, das Kommunikationswege und Inhalte integriert [24]. Die gesundheitsbezogene Nutzung des Internets nimmt laut der Health Information National Trends Survey seit 2003 stetig zu [25]. Digitale Techniken haben hierbei das Arzt-Patienten-Verhältnis bereits heute deutlich verändert. Neben Gesundheitsinformationen spielen digitale Kommunikation und digitale Patientendaten eine wichtige Rolle [26]. Dies führt derzeit zu einer technischen Revolution der Organisation und Erbringung von Gesundheitsdienstleistungen [24]. Das so transformierte Gesundheitswesen wird als eHealth bezeichnet, der informationstechnologische Aspekt als Medizinische Informatik. Der Teilbereich, der sich mit der Interaktion von medizinischen Laien mit elektronischen Gesundheitsdiensten befasst, wird Consumer Health Informatics genannt [27, 28]. Nutzen, Konsequenzen und Nachteile der Digitalisierung in der Medizin werden kritisch diskutiert [29].

Für Patienten sind im Umgang mit dem Internet 7 Aspekte beschrieben, die gesundheitliche Auswirkungen haben können: gut untersucht sind das Finden von Informationen, das Erleben von Unterstützung, die Pflege von Beziehungen, die Beeinflussung von Verhalten und die Inanspruchnahme von Gesundheitsdiensten, während über die Formulierung von Erfahrungen und die Veranschaulichung von Krankheiten bislang wenig bekannt ist [22]. Die digitalen Medien ermöglichen Patienten im Prinzip mehr Mitbestimmung bei Erhalt und Wiederherstellung der eigenen Gesundheit. Diese Entwicklung entsteht in einer Zeit, in der insgesamt die Eigenverantwortung von Patienten zunehmend betont wird [21].

Suchmaschinen machen gesundheitsbezogene Informationen in bisher ungekanntem Ausmaß zugänglich [30]. Es gibt eine noch nicht vollständig verstandene Wechselwirkung zwischen der Einstellung des Nutzers, der Formulierung einer Suchanfrage und dem Ergebnis [31]. Die Identifikation von relevanten und validen Ergebnissen ist dabei wahrscheinlich die größte Herausforderung [30].

Da das Internet eine derart zentrale Rolle im Gesundheitswesen spielt, soll die vorliegende Übersichtsarbeit den aktuellen wissenschaftlichen Kenntnisstand zur Rolle von Gesundheitsinformationen im Internet in der Hals-Nasen-Ohrenheilkunde beschreiben. Neben einer Erörterung der deutlich vergrößerten Zugänglichkeit von Informationen durch das Internet sollen inhaltliche Aspekte von Gesundheitsinformationen im Internet dargestellt werden. Die Auswirkungen der Verfügbarkeit des Internets auf Gesundheitskompetenz und das Arzt-Patienten-Verhältnis sind noch nicht vollständig absehbar, dennoch sollen hier wesentliche Faktoren genannt werden.

\section{Das Internet als Zugangsweg zu Gesundheitsinformationen}

\subsection{Entwicklung der Nutzung des Internets für Gesundheitsinformationen}

Das Internet wird zunehmend von Patienten als Informationsquelle für gesundheitsbezogene Informationen genutzt und hat inzwischen einen enormen Stellenwert erreicht. Diese Entwicklung ist seit einigen Jahren kontinuierlich steigend, sodass davon ausgegangen werden kann, dass inzwischen die große Mehrheit der Patienten das Internet nutzt. Je nach befragter Gruppe und Erhebungszeitpunkt be- richten Patienten in 42-96,4\% der Fälle, dass sie Zugang zum Internet haben [32-34]. 52,3-90,0\% der Patienten nutzen das Internet auch für eine gezielte Suche nach Gesundheitsinformationen [32-36].

Der Stellenwert des Internets im Vergleich zu anderen Informationsquellen ist noch nicht vollständig klar. In den USA geben derzeit 33-70\% der Menschen das Internet als primäre Informationsquelle in Gesundheitsfragen an [25,37]. Auch andere Erhebungen haben konsistent das Internet als bevorzugtes Medium gegenüber Alternativen wie Freunden und Verwandten, gedruckten Medien, Radio und Fernsehen dargestellt $[25,37,38]$. Unter diesen Studien besonders bedeutsam ist die seit 2004 kontinuierlich in den USA durchgeführte Studie Health Information National Trends Survey (HINTS) [39]. Auch in Europa nimmt die Nutzung des Internets zu, ebenso die Bedeutung, die dem Internet relativ zu anderen Medien beigemessen wird [36]. Diese Studien sind jedoch bevölkerungsbezogen und spiegeln möglicherweise nicht die Situation eines Patienten wieder, der mit einer konkreten medizinischen Fragestellung konfrontiert und dabei unmittelbar betroffen ist. Um dies zu klären, sind Untersuchungen der Internetnutzung an Patientenpopulationen erforderlich, die in einer klinischen Einrichtung rekrutiert werden.

Eine erste Studie dieser Art stammt aus dem Jahr 2000 und wurde in einer gastroenterologischen Ambulanz in den USA durchgeführt. Dort wurden 924 Patienten befragt, von denen $50 \%$ (462/924) über Internetzugang verfügten, den 25,4\% (235/924) für gesundheitsbezogene Recherchen nutzten [40]. Auch der Zugang zum und der Stellenwert des Internets bei Patienten der Hals-Nasen-Ohrenheilkunde wurde seitdem bereits mehrfach untersucht. Auch hier zeigt sich eine zunehmende Nutzung des Internets. Dies betrifft den Internetzugang, der noch 2002 mit 45,5\% angegeben wurde [41], während in der jüngsten vorliegenden Untersuchung aus 2018 ein Wert von 91,3\% erreicht wurde [42]. Eine Studie verglich direkt $2 \mathrm{Er}$ hebungen von 2000 und 2006 und stellte dabei eine deutliche Steigerung von Internetzugang und gesundheitsbezogener Nutzung dar [43]. Im Gegensatz zu bevölkerungsbezogenen Erhebungen wurde bisher in keiner Studie mit Patienten aus klinischen Einrichtungen das Internet als wichtigste Quelle für Gesundheitsinformationen dargestellt. Wenn eine Priorität erhoben wurde, war das Internet eine nachrangige $[41,44-46]$ oder sogar die am wenigsten relevante Informationsquelle $[47,48]$. Wichtiger als das Internet waren dabei der behandelnde HNO-Arzt $[44,45,47,48]$, der behandelnde Kinderarzt $[45,47,48]$, der Hausarzt $[41,44,46,48]$, andere behandelnde Ärzte [46], nichtärztliche Berufsgruppen aus dem Gesundheitswesen (Zahnarzt, Apotheker, Krankenpflege usw.) [45, 47, 48], Informationsbroschüren $[41,45,47,48]$, Freunde oder Verwandte $[41,44,45,47,48]$, Bücher [41, 47, 48] sowie Zweitmeinungen [44]. Möglicherweise treten also bei konkreter eigener Betroffenheit doch wieder die herkömmlichen Informationsquellen in den Vordergrund.

Auffällig ist, dass die bisherigen Untersuchungen zur gesundheitsbezogenen Internetnutzung im Bereich der Hals-Nasen-Ohrenheilkunde noch methodische Schwächen aufweisen. So verwenden die Studien häufig eigene, nicht validierte Fragebögen [41-44, 46,49 ]. Nur in einer Untersuchung [50] wurde ein zuvor publizierter, getesteter Fragebogen verwendet [40], weitere Untersuchungen $[45,47,48]$ adaptierten früher verwendete Fragebögen $[41,47,50,51]$. Die Situation in Deutschland wurde bisher erst in einer Studie untersucht [44]. Eine Übersicht zu Untersuchungen zur 
Internetnutzung von HNO-Patienten in klinischen Kohorten findet sich in $>$ Tab. 1.

\section{2 technische Aspekte bei Gesundheitsrecherchen durch Laien}

Verschiedene Besonderheiten zeichnen eine gesundheitsbezogene Internetrecherche durch Laien aus. Zum einen ist die Suchstrategie häufig wenig optimal, weil diese üblicherweise wenig von den zahlreichen Möglichkeiten der Suchmaschinen Gebrauch machen um Suchanfragen zu verfeinern oder einzuschränken [52,53]. Beispielsweise wird eine Suche über Schlagworte häufiger verwendet als eine Suche nach Kategorien [54]. Zum anderen nutzen Laien in der Regel nur allgemeine Suchmaschinen wie bspw. Google und nahezu nie medizinische Portale [53]. Überwiegend werden nur wenige Treffer vom Beginn der Suchergebnisse weiter verfolgt $[53,54]$, in weniger als $3 \%$ der Fälle werden Treffer nach den ersten 10 Rängen angeklickt [52]. Oft können sich die Nutzer nach einer Recherche nicht erinnern, von welcher Internetseite eine bestimmte Information stammt [52].

Auf der Suche nach Gesundheitsinformationen verbringen Probanden relativ viel Zeit damit, die Vertrauenswürdigkeit von Suchergebnissen zu beurteilen [54]. Allgemein werden Internetseiten öffentlicher Einrichtungen, ein professionelles Layout, verständliche Fachsprache und der Verweis auf wissenschaftliche Quellen als besonders vertrauenswürdig angesehen [52]. Möglicherweise gibt es dabei jedoch nationale Unterschiede [53]. Bestrebungen, vertrauenswürdige Internetseiten zu priorisieren bestehen in der Entwicklung einer für den Gesundheitsbereich spezialisierten Suchmaschine mit dem Namen „Khreshmoi für alle“ (Khresmoi for everyone, K4E) [55]. Neben der wahrscheinlich noch äußerst geringen Bekanntheit präferieren Probanden im direkten Vergleich dennoch Google, was die Notwendigkeit einer verstärkten Gesundheitsaufklärung vermuten lässt [56].

\subsection{Nachteile von Gesundheitsinformationen im Internet}

Nachteile der durch das Internet stark gestiegenen Verfügbarkeit von Gesundheitsinformationen werden regelmäßig diskutiert. So wurde die Cyberchondrie (auch als Morbus Google bezeichnet) als übersteigerte Angst um den eigenen Gesundheitsstatus auf der Grundlage von Gesundheitsinformationen aus dem Internet beschrieben [57-59]. Problematisch kann es auch sein, wenn Patienten aufgrund von Informationen aus dem Internet z. B. Vorsorgeuntersuchungen nicht in Anspruch nehmen [60]. Anekdotische Informationen aus dem Internet bergen weiterhin das Risiko, dass eindrücklich illustrierte Fälle mit falschen oder fehlleitenden Informationen verknüpft sind [22]. Eine systematische Auswertung ergab bisher allerdings wenige publizierte Fälle, bei denen es zu Schäden nachweislich durch Gesundheitsinformationen aus dem Internet kam [61]. Wahrscheinlich sind die Auswirkungen daher eher auf der Ebene des Arzt-Patienten-Verhältnisses zu suchen.

Im Bereich der Hals-Nasen-Ohrenheilkunde wurde der Nutzen eines Algoritmus, der auf einer Internetseite zur Verfügung gestellt worden war, für die Diagnosestellung untersucht. Während in einer Patientengruppe von 61 Frauen und Männern bei 70,5\% (43/61) durch die Anwendung eine korrekte Diagnose ermittelt wurde, war dies allerdings nur eine von im Durchschnitt 13 angegebenen Differentialdiagnosen. Nur in 16,4\% (10/61) der Fälle war die erste vor-

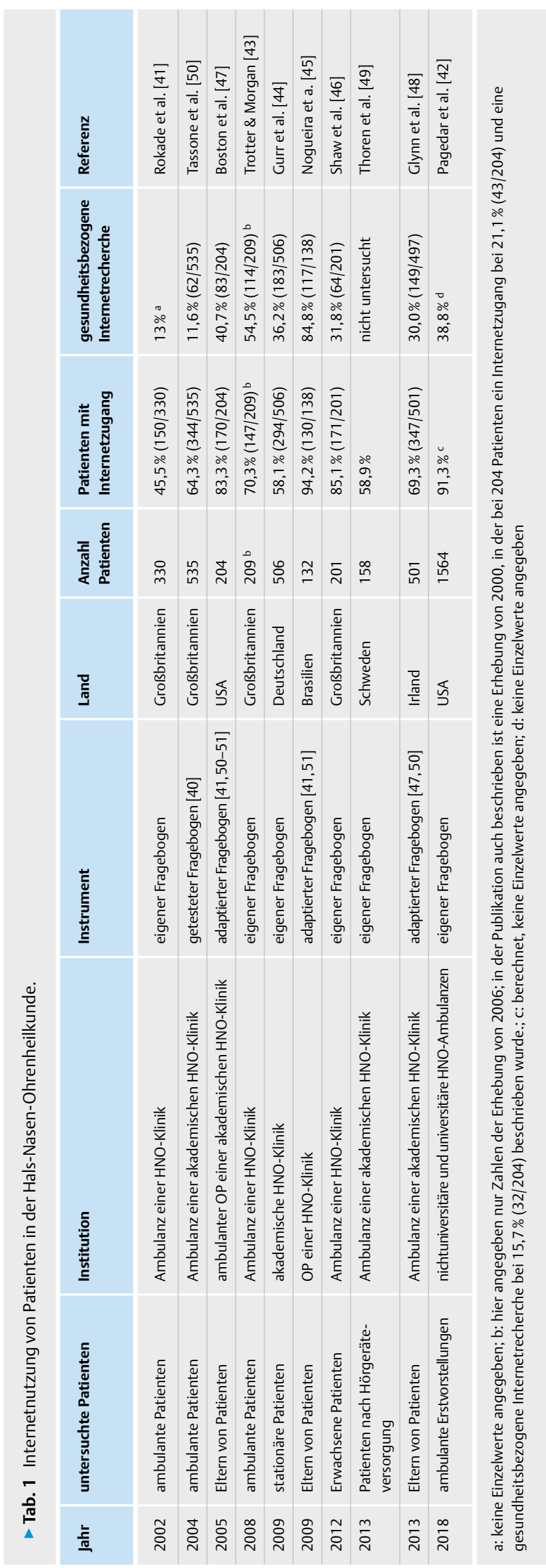


geschlagene Diagnose korrekt [62]. Diese Untersuchung illustriert häufige Probleme mit Internetanwendungen, die zwar über eine breite Wissensbasis verfügen, aber in der Regel ohne menschlichen medizinischen Sachverstand und klinische Erfahrung von eingeschränktem Nutzen sind.

\section{Qualität von Gesundheitsinformationen im Internet}

Mit Feststellung der Tatsache, dass Patienten zunehmend das Internet für gesundheitsbezogene Recherchen nutzen, stellt sich naturgemäß die Frage nach der Beschaffenheit von Gesundheitsinformationen im Internet. Hierbei wird insbesondere die Qualität und inhaltliche Richtigkeit häufig in Frage gestellt $[63,64]$. Patienten scheinen bei gesundheitlichen Erfahrungen, die sie nicht dem eigenen Erfahrungsschatz zuordnen können, möglicherweise primär an Beispielen von Erfahrungen und Entscheidungen anderer Menschen interessiert zu sein [22]. Allerdings sind faktenbasierte Gesundheitsinformationen im Internet deutlich detaillierter untersucht worden, da deren Qualität schon länger diskutiert wird [63].

Die Beurteilung der Qualität von Gesundheitsinformationen im Internet hängt vom betrachteten Nutzerkreis ab, da bspw. für Patienten und Laien die Lesbarkeit im Vordergrund stehen wird, während Fachkreise eher wissenschaftliche Belege erwarten [64]. Nahezu immer sind Gesundheitsinformationen für den Laien zu schwierig lesbar [65]. Zudem gibt es noch keinen Konsens in Bezug auf die Bewertungsmaßstäbe und viele bisherige Studien weisen methodische Schwächen bei insgesamt unzureichender Qualität auf [66]. Ein hoher Schwierigkeitsgrad bei der Lesbarkeit von Patienteninformationen führt dazu, dass ein großer Teil der Patienten daraus nur geringen oder keinen Nutzen ziehen kann [67]. Möglicherweise sind dabei Lesbarkeit und Vollständigkeit Gegensätze [68].

\subsection{Methoden}

Es gibt Bestrebungen, die Qualität von Gesundheitsinformationen im Internet systematisch zu erfassen und zu verbessern. Neben den inhaltlichen Aspekten, die naturgemäß fachspezifisch zu beurteilen sind, wird allgemein die Lesbarkeit als wichtiger Gesichtspunkt erachtet. Gesundheitsinformationen sind in der Regel aus spezifischen Gründen schwieriger zu verstehen als alltägliche Texte. Dies kann selbst für sprachlich sehr kompetente Laien eine Hürde sein [69, 70]. Empfohlen wird bezüglich der Lesbarkeit bspw. vom Gesundheitsministerium der USA (Department of Health and Human Services), dass die Schwierigkeitsstufe dem Niveau maximal der 7. Jahrgangsstufe des Bildungssystems der USA entspricht [71]. Die Lesbarkeit von Texten kann linguistisch mittels Formeln dargestellt werden, indem bspw. die durchschnittliche Anzahl von Silben pro Wort, die Anzahl von Wörtern pro Satz sowie der Anteil der allgemeinverständlichen Wörter oder der Wörter mit 3 oder mehr Silben erfasst wird $[72,73]$. Das Ergebnis einer solchen Formel kann ein generischer Index sein oder ein Zahlenwert, welcher der Jahrgangsstufe des amerikanischen Bildungssystems entspricht [74].

Eine weit verbreitete Formel ist der Flesch Reading Ease Score. Dieser ist eine dimensionslose Verhältniskennzahl, die sich aus der Anzahl der Sätze, Wörter und Silben in einem Text ergibt und in der Regel Werte zwischen 0 und 100 annimmt. Hierbei stellen höhere
Zahlenwerte eine einfachere Lesbarkeit dar [75]. Der Vergleichsmaßstab ist auf die Gegebenheiten der englischen Sprache angepasst, es existiert allerdings auch eine modifizierte Formel für deutsche Texte [76]. Das Flesch-Kincaid-Grade-Level ist ein Wert, der dem Kompetenzstand einer Jahrgangsstufe des US-amerikanischen Bildungssystems entspricht [77]. Ausgangswerte sind die durchschnittliche Satzlänge und die durchschnittliche Silbenzahl pro Wort. Weitere regelmäßig verwendete Lesbarkeitsformeln nach ähnlichem Prinzip sind Gunning Frequency of Gobbledygook (Gunning-Fog index) [78] und Simple Measure of Gobbledygook (SMOG) [79].

Die Lesbarkeitsforschung für Gesundheitsinformationen beschränkt sich bisher nahezu ausschließlich auf die englische Sprache und es liegen nur einzelne für die deutsche Sprache angepasste Lesbarkeitsindizes vor $[76,80]$. Daneben findet sich in deutschen Untersuchungen auch ein primär deutsches Instrument wie der Lesbarkeitsindex LIX [81].

Die Qualität von Gesundheitsinformationen kann auch anhand von definierten Kriterien systematisch erfasst und beschrieben werden. Im Journal of the American Medical Association wurden 4 solche Kriterien formuliert. Dabei wird die deutliche und vollständige Offenlegung von Autorschaft und Finanzierung, der Nachweis von Quellen und Referenzen sowie der Zeitpunkt von Erstellung und Überarbeitungen gefordert [63]. Daneben existieren ausführlichere Instrumente als Fragebogen, welche es ermöglichen, die Qualität systematisch zu beurteilen. Bedeutend und auch in deutscher Sprache verfügbar ist der validierte DISCERN-Fragebogen [82-86]. Dieser besteht aus 8 Fragen zur Zuverlässigkeit der Publikation (Ziele, Quellen, Ausgewogenheit), 7 Fragen zu Behandlungsalternativen (Wirkungsweise, Nutzen, Risiken) und einer Frage zur Gesamtbewertung [82].

Zuletzt gibt es Bestrebungen, mittels eines Zertifizierungsverfahrens die Qualität von Gesundheitsinformationen im Internet für den Leser sichtbar zu machen. Am bekanntesten ist dabei die Health on the Net (HON)-Stiftung, die einen Verhaltenskodex für Internetseiten entwickelt hat [87]. Dieser Kodex beinhaltet allgemeine Kriterien wie die Anforderung nach Kontaktdaten und Datierung des Materials sowie Datenschutzanforderungen und Beurteilung der Qualifikation der Urheber. Zertifizierungen nach diesem Kodex existieren nun seit über 20 Jahren. In diesem Zeitraum wurden über 20000 lnternetseiten untersucht, etwa $13 \%$ der evaluierten Internetseiten werden seit über 10 Jahren kontinuierlich re-zertifiziert [55, 88]. Es gibt Überlegungen, dieses Zertifikat automatisiert bei Suchanfragen zu berücksichtigen $[89,90]$. Der gewählte Ansatz führt sicher zu guter Vergleichbarkeit und hoher Validität des Zertifikats, schließt jedoch aufgrund der Herangehensweise eine auch nur annähernd vollständige Abdeckung des Internets aus.

Eine Sonderstellung nimmt MedlinePlus (http://www.medlineplus.gov) ein. Dies ist ein kostenfreier Informationsdienst der United States National Library of Medicine (NLM), der an die Allgemeinbevölkerung gerichtet ist. Ausgehend von Nutzerstatistiken der NLM wurde ein Katalog von über 1000 Erkrankungen erstellt, der kurze Zusammenfassungen mit einer Linksammlung verknüpft. Daneben gibt es ein Fachwörterbuch mit kurzen Definitionen zu Erkrankungen, ein Adressverzeichnis von Gesundheitseinrichtungen und Angehörigen der Heilberufe in den USA sowie Linksammlungen zu professionellen Organisationen, Publikationen, Bibliotheken und weiteren Wörterbüchern. Diese Inhalte müssen jedoch von einer Redaktion zusammengestellt und regelmäßig aktualisiert werden [91]. 
Dies ist ein sehr aufwendiges Verfahren, das dazu führt, dass jeweils immer nur ein kleiner Bruchteil der im Internet verfügbaren Gesundheitsinformationen über MedlinePlus bewertet und erreichbar ist [55]. Aus deutschsprachiger Sicht ist es sicherlich auch ein Nachteil, dass MedlinePlus mit Ausnahme von wenigen Einzelinformationen ausschließlich auf Englisch und Spanisch verfügbar und zudem darauf ausgerichtet ist, die Verhältnisse im Gesundheitswesen der USA abzubilden.

\subsection{Untersuchungen}

Gesundheitsinformationen im Bereich der Hals-Nasen-Ohrenheilkunde werden im Internet von vielen verschiedenen Anbietern zur Verfügung gestellt. Spezifische Untersuchungen liegen bisher zu nicht-universitären Kliniken in Deutschland [92] sowie zu universitären HNO-Kliniken in Deutschland [93] und den USA [94] vor. Daneben wurden in den USA die Seiten der Fachgesellschaften aus dem Gebiet der HNO und verwandter Fachgebiete [95-100] analysiert. Eine separate Auswertung betrachtete die Inhalte aus der pädiatrischen HNO auf Internetseiten von Krankenhäusern und der Fachgesellschaft der USA [101].

Einhellig wurden die verfügbaren Patienteninformationen als für die Allgemeinheit zu schwierig eingestuft. Der Schwierigkeitsgrad von Informationsmaterialien auf den Internetseiten aller Amerikanischen Fachgesellschaften aus dem Gebiet der Hals-Nasen-Ohrenheilkunde [95-97] und benachbarter Gebiete [98, 100] übersteigt das empfohlene Niveau. Dies trifft auch auf die spanischsprachigen Informationen zu, die Institutionen in den USA auf ihren Internetseiten vorhalten [99]. Auch die überwiegende Zahl der institutionellen Internetseiten zur pädiatrischen Hals-Nasen-Ohrenheilkunde ist für den durchschnittlichen amerikanischen Bürger zu schwierig [101]. Dieser allgemeine Trend betrifft jedoch nicht nur die HNO alleine, auch im Vergleich verschiedener chirurgischer Spezialgebiete wurde festgestellt, dass alle im Internet verfügbaren Informationen der USamerikanischen Fachgesellschaften zu schwierig lesbar sind. Die HNO-Gesellschaft der USA liegt dabei im Mittelfeld [102]. Konsequenzen aufgrund dieser allseits berichteten Diskrepanz zwischen den Anforderungen der verfügbaren Gesundheitsinformationen und der durchschnittlichen Lesekompetenz der Patienten werden diskutiert [67] und gehen so weit, dass grundsätzlich hinterfragt wurde, ob Krankenhäuser Gesundheitsinformationen im Internet zur Verfügung stellen sollen [92].

Gesundheitsinformationen, die auf den Internetseiten von Krankenhäusern oder Fachgesellschaften dargestellt sind, stellen sicher einen wichtigen Bereich für die Außendarstellung der Institution dar. Jedoch wird wahrscheinlich nur ein kleiner Teil der Patienten gezielt solche Seiten auswählen. Deutlich bedeutender bei der Erschließung des Informationsangebots im Internet sind Suchmaschinen. Dementsprechend nimmt die Analyse von Gesundheitsinformationen, die über Suchmaschinen aufgefunden werden können, mehr Bedeutung in der wissenschaftlichen Literatur dazu ein.

Für das Gebiet Hals-Nasen-Ohrenheilkunde haben 21 Untersuchungen zu Krankheitsbildern und 18 zu Prozeduren die Suche durch Laien nach Gesundheitsinformationen im Internet simuliert und die Qualität der resultierenden Ergebnisse untersucht. Die Themen Onkologie [103-108], Audiologie/Neurootologie/Otologie [109-117] und plastische Chirurgie [118-122] waren hierbei besonders stark vertreten. Das Schrifttum wird aber bisher von Untersuchungen zu englischsprachigen Gesundheitsinformationen dominiert. Darüber hinaus wurden in jeweils einzelnen Studien spanisch- [105] und türkischsprachige Inhalte untersucht [123].

In allen bisherigen Studien wurde Google verwendet. Zusätzlich kamen Bing [103, 109, 113, 114, 118, 124-127], Yahoo [103-105, 109, 113, 114, 118, 124-130], HONsearch [104], MedlinePlus [105], MSN, AOL und Ask Jeeves [130] sowie omnimedicalsearch.com, pogofrog.com, searchmedica.co.uk und imedisearch.com [125] zum Einsatz. Die Lesbarkeit des gefundenen Materials wurde durch den Flesch Reading Ease Score mit 29,7-61,5 angegeben [103, 104, 106109, 112, 114, 115, 118, 122, 126, 130-139]. Dies entsprichthauptsächlich den Bereichen, die als schwer (difficult) oder ziemlich schwer (fairly difficult) eingestuft werden (Punktwert 30-50 bzw. 50-60) und üblicherweise in akademischen oder anspruchsvollen Texten Anwendung finden [75]. Diese Anforderungen an die Lesbarkeit übersteigen die durchschnittliche Lesekompetenz der durchschnittlichen Bevölkerung deutlich. Mit dem DISCERN-Instrument wurden Summenwerte von 28,1-57 erreicht $[103,109,111,113,114,118,122-124,126,136$, 139]. Dieser Fragebogen versucht systematisch die Verlässlichkeit und den Nutzen eines Textes als Patienteninformation zu erfassen. Die erreichten Werte entsprechen einem deutlichen Abstand zum optimalen Punktwert von 80 , sodass dies auf eine allenfalls mittlere Qualität bei den im Internet verfügbaren Informationen schließen lässt. Ein Health on the net- (HON-) Zertifikat lag bei den untersuchten Internetseiten nur mit einem relativ niedrigen Anteil von $0-30,6 \%$ vor $[103,111,122$, 133, 134, 136, 138]. Eine Übersicht von Studien zu Krankheitsbildern aus der Hals-Nasen-Ohrenheilkunde findet sich in $>$ Tab. 2, eine Übersicht von Studien zu Prozeduren in > Tab. 3.

Eine systematische Übersichtsarbeit hat sich mit Gesundheitsinformationen zum Thema Hören befasst. Die Auswertung von 8 Studien bestätigte eine geringe Lesbarkeit des untersuchten Materials, dessen Schwierigkeitsgrad weit über den Empfehlungen lag. Als Konsequenz wurde eine systematische Berücksichtigung einer guten Lesbarkeit bei der Erstellung von medizinischem Informationsmaterial gefordert [140].

\section{3 soziale Medien}

Soziale Medien sind die charakteristischen Anwendungen des Web 2.0 [11]. Die Erstellung und Verbreitung von Inhalten erfolgt durch die Verknüpfung von Nutzern und macht neue Wege der Informationsverteilung und neue gesundheitsbezogene Anwendungen möglich [141]. Partizipation, Datenmanagement und kollektive Intelligenz sind auch Merkmale gesundheitsbezogener Anwendungen [142]. Aus Patientensicht steht der Aspekt des Erfahrungsaustausches im Vordergrund [22], daneben werden so gesundheitsbezogene Computerspiele, mobile Anwendungen und eine leichte Verbreitung von Videos möglich [143]. Die Bedeutung sozialer Medien bei der Suche nach Gesundheitsinformationen nimmt kontinuierlich zu und es wird erwartet, dass sich soziale Medien im professionellen Alltag praktizierender Mediziner weiter durchsetzen [144, 145]. Bisherige Anwendungsbereiche sozialer Medien im Gesundheitswesen beinhalten die Vereinfachung und Beschleunigung der Kommunikation zwischen Patienten und zwischen Ärzten und Patienten [16, 146]. Junge, technikaffine Menschen sind wie bei anderen technischen Neuerungen die ersten, die von gesundheitsbezogenen sozialen Medien profitieren. Potentielle Nutzer sind darüber hinaus aber auch solche mit niedrigem sozioökonomischem Status und chronisch Kranke [143]. 


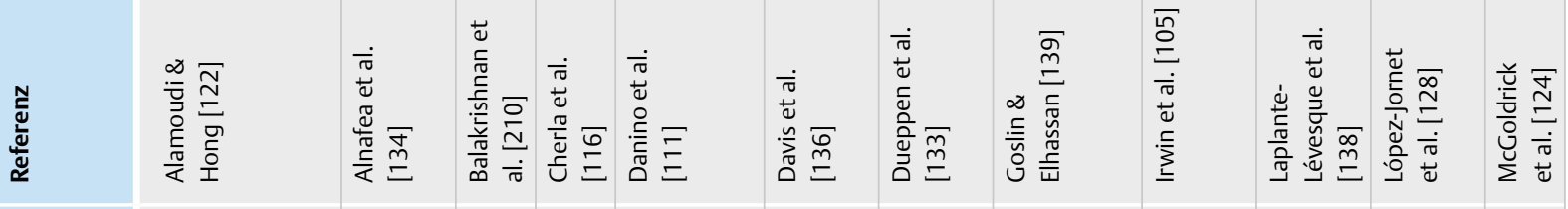

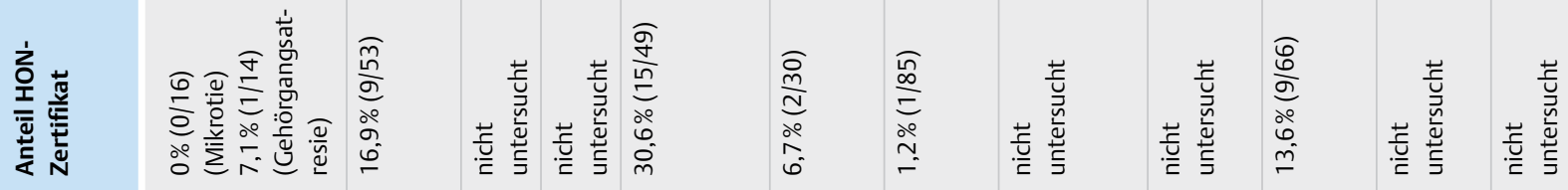

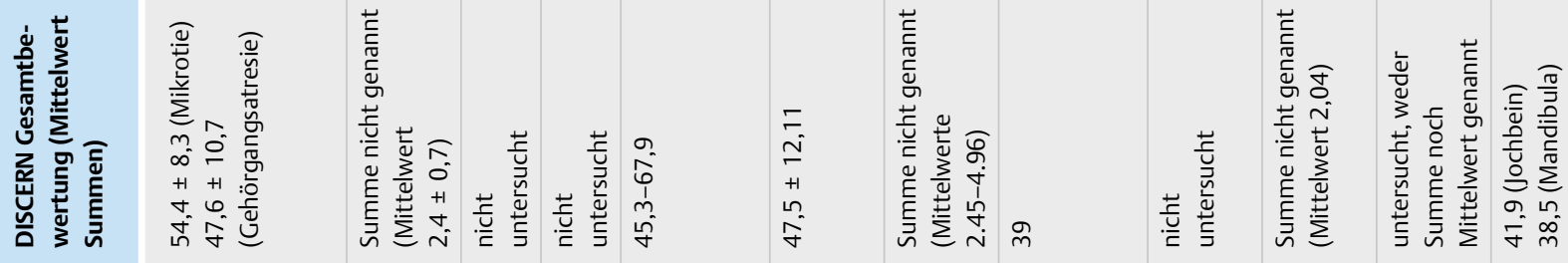

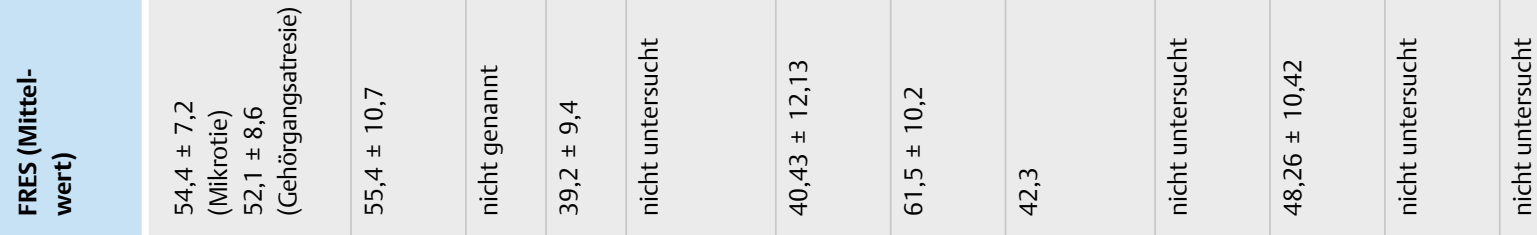

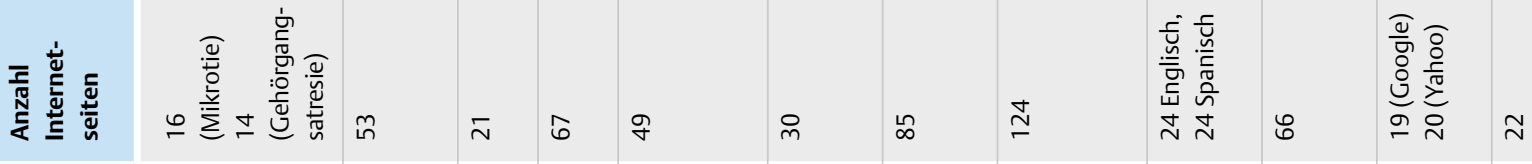

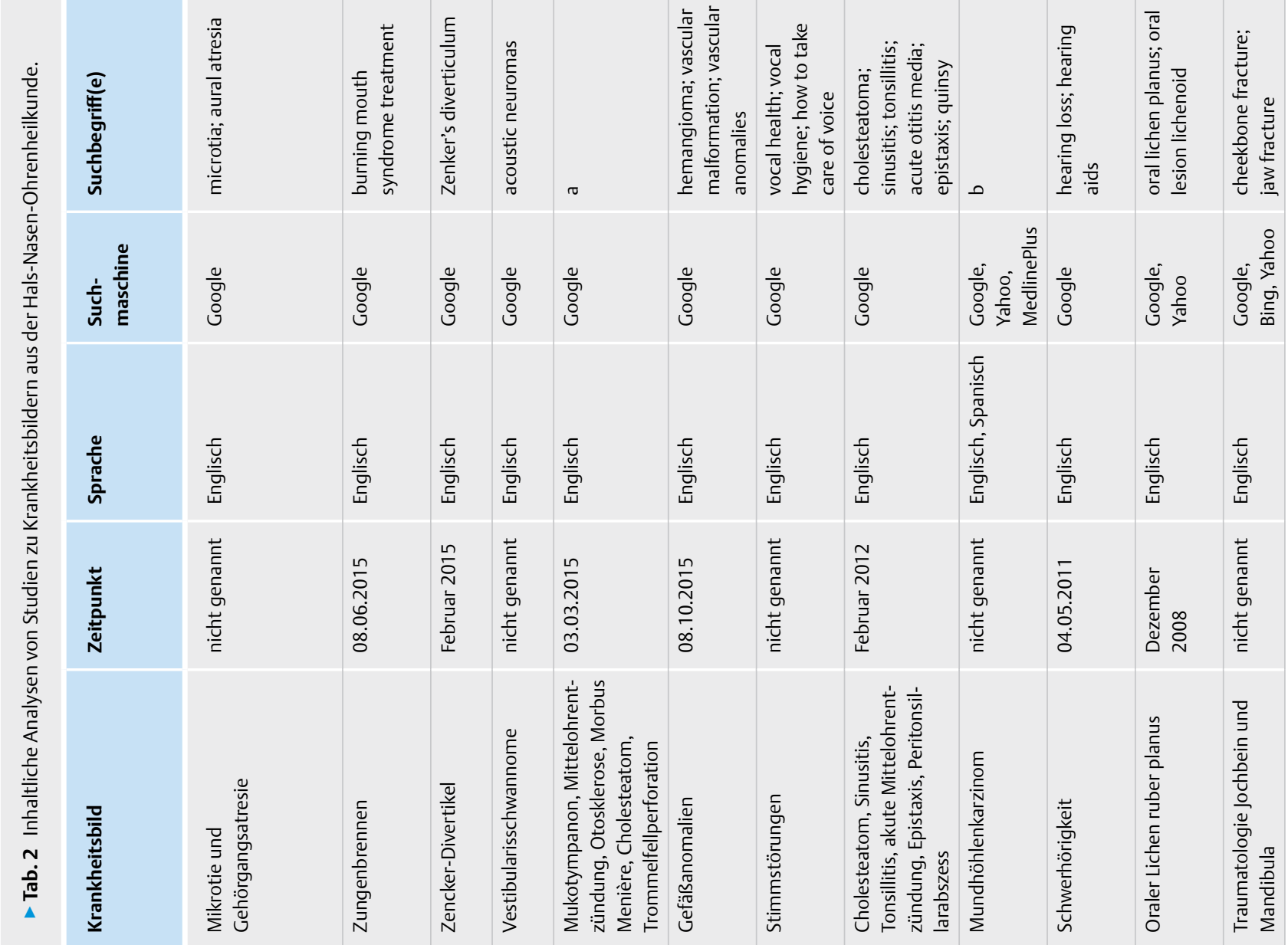




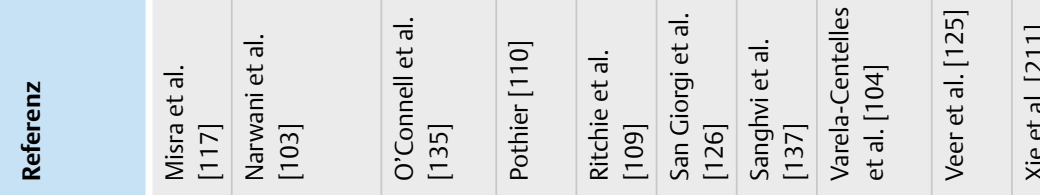

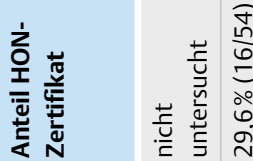

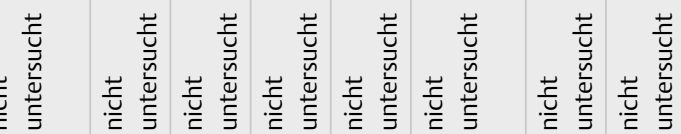

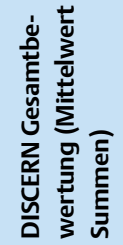

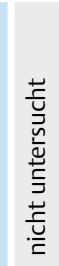

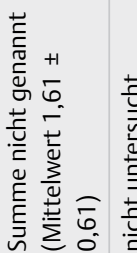

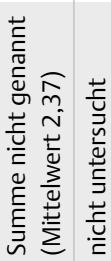

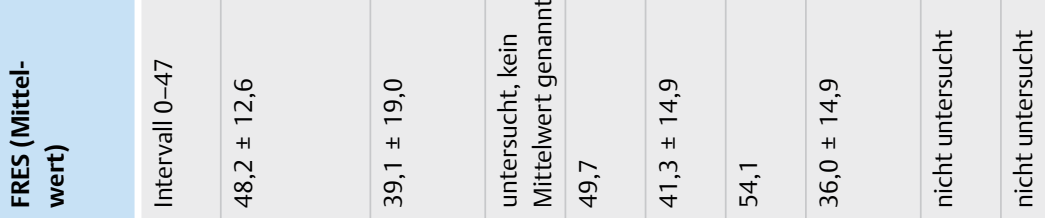

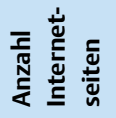

$\stackrel{+}{\circ}$

เก

ำ โ

$\because \approx$

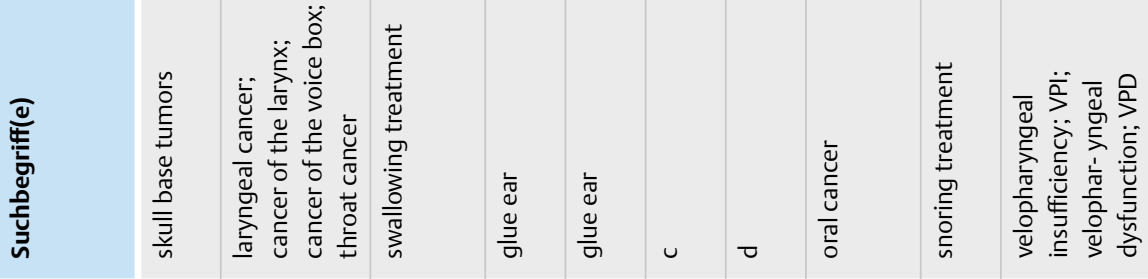

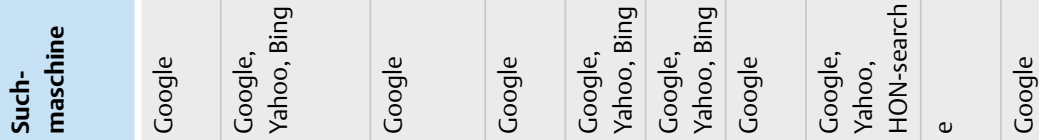

을

टें

产

范方

产

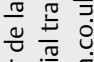

苋

نั

뜨

동

产苋

㐫突

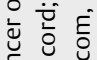

厄 ত

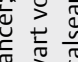

范

동

造

记

壱 음

घั

등

뜽

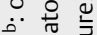

ㅎ⿺ㄴ 高皆

ฉ

先盛

ชั

웅

荇苋

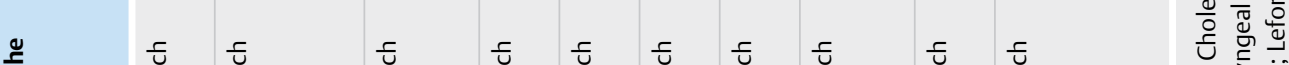

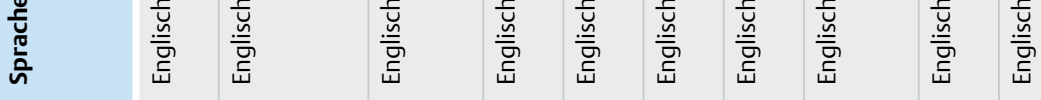

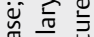

剀

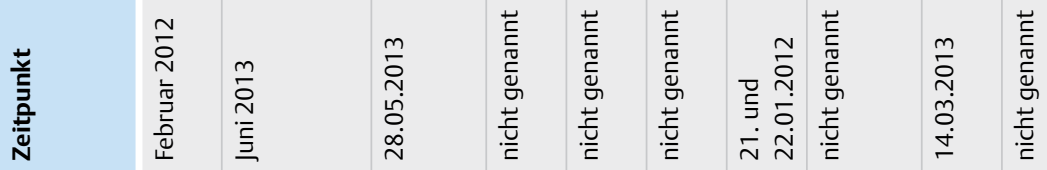

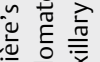

恼 $\frac{0}{\overline{\bar{a}}} \stackrel{\overline{\bar{x}}}{E}$

$\sum_{i \frac{\pi}{n}} \frac{\pi}{\pi}$

要

苍 든

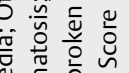

E

产苍岂

ㄴㄷㄴ

过 웜

光产言至 


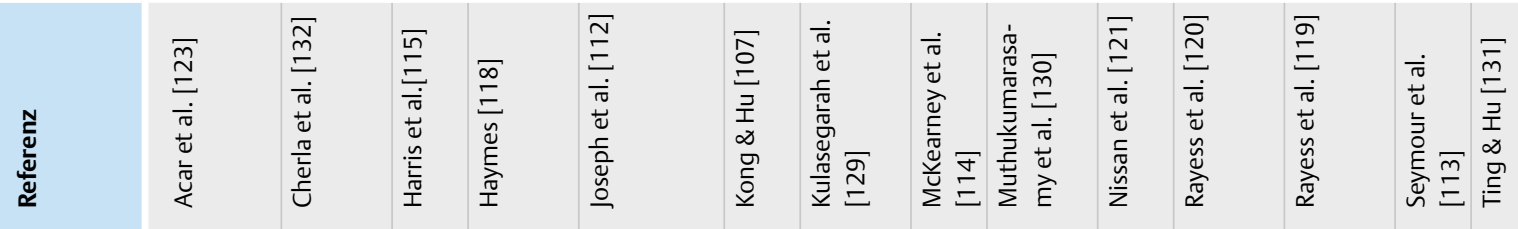
IIn
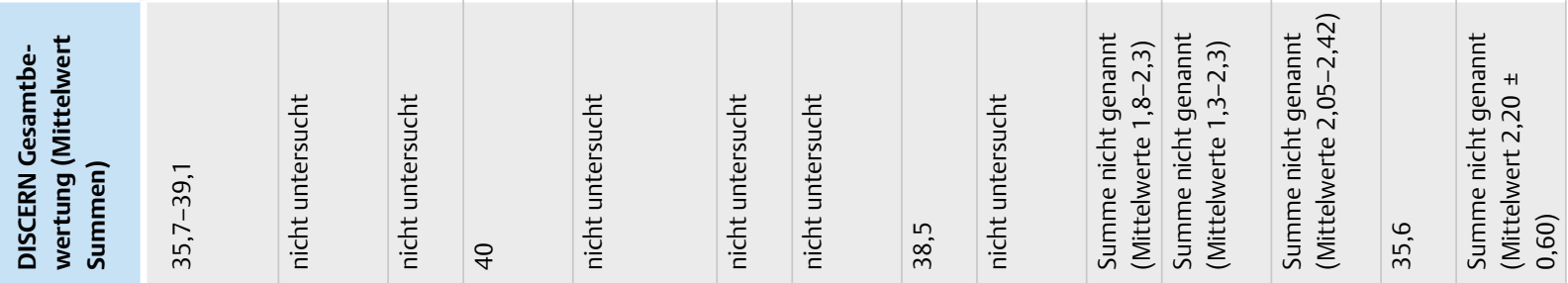

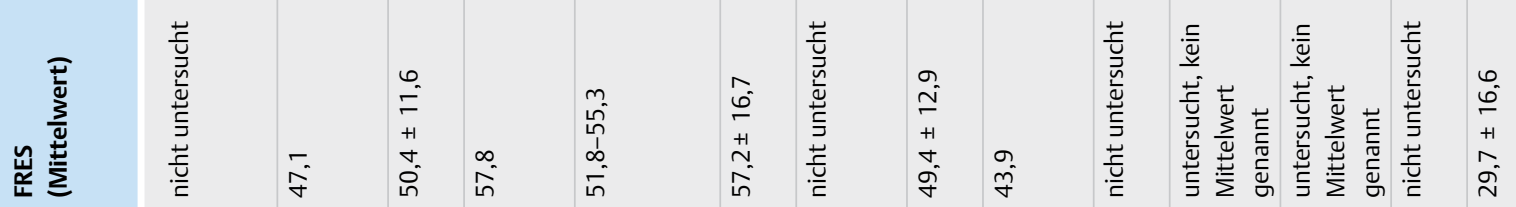

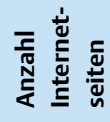

$8 \quad \bar{m} \quad \circ$

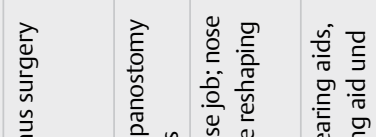

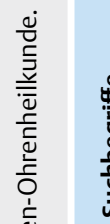

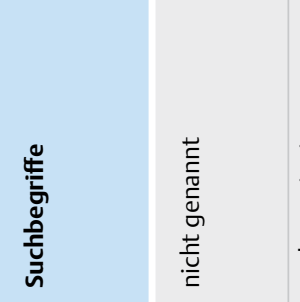

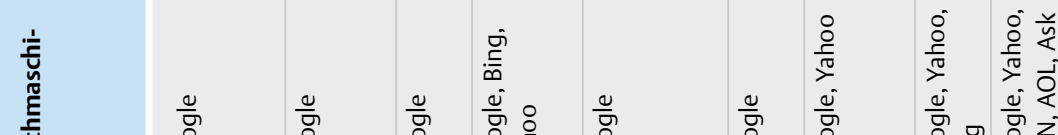

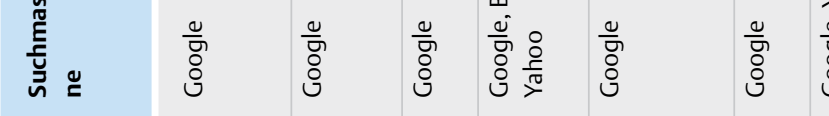

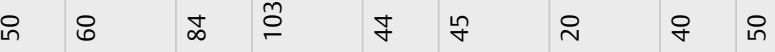

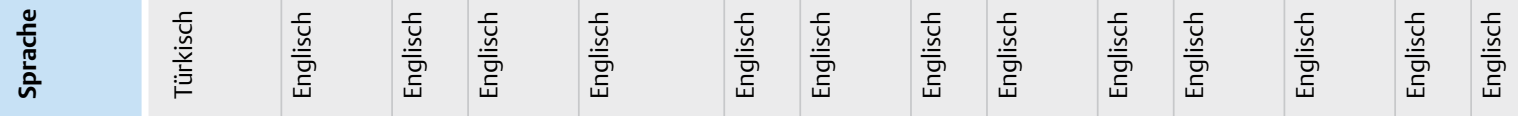

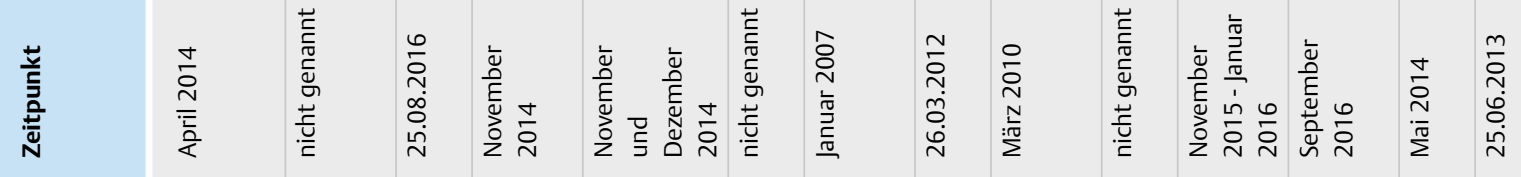




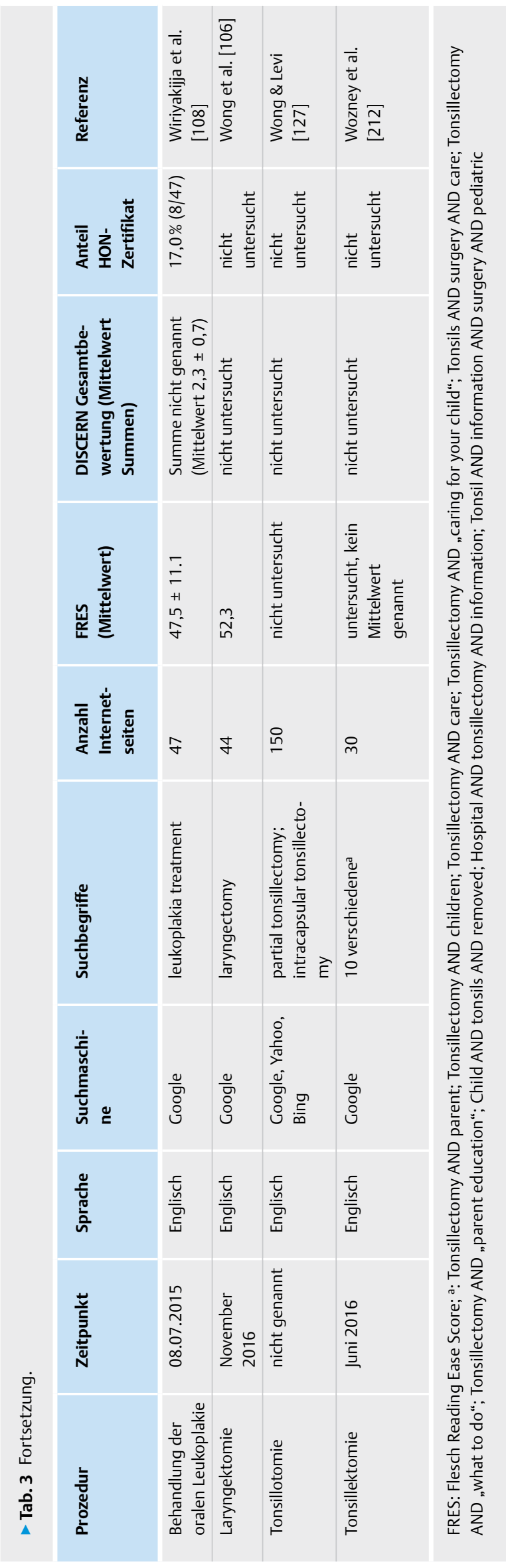

In vielen Bereichen gibt es Bedenken gegenüber dem Nutzen sozialer Medien im Gesundheitswesen u. a. in Bezug auf Datenschutz und professionelles Verhalten [16]. Daher hat bspw. das American College of Physicians (ACP), die US-amerikanische Fachgesellschaft für Innere Medizin, einen sehr zurückhaltenden Verhaltenskodex für das professionelle Auftreten in sozialen Medien verabschiedet [147]. Als Nachteile werden dabei angeführt, dass eine Überlegenheit sozialer Medien über herkömmliche Kommunikationswege nicht wissenschaftlich gesichert ist, dass Datenschutzbedenken bestehen und dass neue Technologien von eigentlichen medizinischen Problemen ablenken könnten [148]. Während somit erhebliche Risiken gesehen werden, gibt es auch Stimmen, nach denen die Vorteile die Nachteile überwiegen [145].

Für die Hals-Nasen-Ohrenheilkunde wurden vielfältige Einsatzmöglichkeiten sozialer Medien besonders in Hinblick auf eine Verbesserung eines kontinuierlichen Arzt-Patienten-Kontakts beschrieben. Dies könnte dazu beitragen, dass Patienten von nicht vertrauenswürdigen Internetseiten ferngehalten werden. Mit Twitter, Facebook und Blogspot können Nachrichten unterschiedlicher Länge an einen Nutzerkreis gesendet werden, welche dem Autor folgen (Follower). YouTube stellt eine Plattform zur Verfügung, welche die nutzerfreundliche Abrufbarkeit von Videos gewährleistet. Ärzte könnten über soziale Medien Informationen verbreiten, die häufig von Patienten benötigt werden. Dies würde dann einen zielgerichteteren und effizienteren persönlichen Kontakt ermöglichen. Darüber hinaus bietet der Kontakt zum Arzt über soziale Medien für Patienten den Zugang zu einer vertrauenswürdigen Informationsquelle [146].

Durch die hohe Geschwindigkeit und die Möglichkeit der Weiterverbreitung von Informationen über Re-Tweets kann Twitter für Einrichtungen und Personen, die im Gesundheitswesen tätig sind, ein effizientes Kommunikationsmedium darstellen [149]. Kritisch gesehen wird neben dem Zeitaufwand, den ein weiterer Kommunikationskanal beansprucht, ein hoher Anteil an Fehlinformationen sowie die für das Internet typische Schwierigkeit, die Vertrauenswürdigkeit einer Quelle festzustellen [150]. Für die Hals-Nasen-Ohrenheilkunde gibt es bisher keine systematische Übersicht zur Nutzung und zum Nutzen von Twitter. Eine beispielhafte Übersicht mit einer Auswahl an Twitter-Accounts im Bereich der HNO-Heilkunde findet sich in > Tab. 4.

Als eine der am meisten frequentierten Internetseite überhaupt spielt Wikipedia auch für Gesundheitsinformationen eine wichtige Rolle. Wahrscheinlich enthält Wikipedia mehr als 164000 Artikel zu Gesundheitsthemen, die weltweit mehr als 10000000 mal gelesen werden [151]. Artikel aus Wikipedia werden bei Suchergebnissen oft an prominenter Stelle aufgeführt $[152,153]$ und neben Patienten auch von Ärzten, Angehörigen anderer Gesundheitsberufe sowie Medizinstudenten häufig genutzt $[151,154]$. Während sehr wenige inhaltliche Fehler nachzuweisen sind [16], ist die Lesbarkeit von Wikipedia-Artikeln allerdings tendenziell sogar schlechter als bspw. Informationen von öffentlichen Einrichtungen [152]. Eine weitere Schwäche von Wikipedia ist, dass mögliche Interessenskonflikte von Autoren noch nicht berücksichtigt oder offengelegt werden können [16]. Der Nutzen von Gesundheitsinformationen bei Wikipedia für Patienten und die Auswirkungen auf das Arzt-Patienten-Verhältnis wurden bisher noch nicht systematisch untersucht [153]. Neben Wikipedia gibt es zahlreiche weitere auf Gesundheitsthemen spezialisierte Wikis, die jedoch an Artikeln und Nutzern weit hinter Wikipedia zurück bleiben [152]. 
- Tab. 4 Auswahl und Aktivität von Twitter-Accounts mit Bezug zur Hals-Nasen-Ohrenheilkunde.

\begin{tabular}{|c|c|c|c|c|c|c|}
\hline Nutzername & Nutzer & Art & Land & $\begin{array}{l}\text { Follo- } \\
\text { wer }\end{array}$ & Tweets & $\begin{array}{l}\text { bei Twitter } \\
\text { aktiv seit }\end{array}$ \\
\hline @phonak & Phonak (Sonova Holding AG) & Medizintechnikunternehmen & Schweiz & 16583 & 10650 & 26.11 .2012 \\
\hline @drluebbers & $\begin{array}{l}\text { Dr. Christian Lübbers, } \\
\text { Weilheim i. OB }\end{array}$ & niedergelassener HNO-Arzt & Deutschland & 11704 & 8053 & 18.02.2015 \\
\hline @AcademyofAuD & $\begin{array}{l}\text { American Academy of } \\
\text { Audiology }\end{array}$ & Fachgesellschaft für Audiologie & USA & 11301 & 5459 & 09.01.2009 \\
\hline @AAOHNS & $\begin{array}{l}\text { American Academy of } \\
\text { Otolaryngology-Head and } \\
\text { Neck Surgery (AAO-HNS) }\end{array}$ & $\begin{array}{l}\text { Fachgesellschaft für Hals-Nasen- } \\
\text { Ohrenheilkunde }\end{array}$ & USA & 7892 & 4810 & 23.03.2009 \\
\hline @medel & MED-EL GmbH & Medizintechnikunternehmen & Österreich & 6070 & 2173 & 02.02 .2010 \\
\hline @JAMAOto & $\begin{array}{l}\text { JAMA Otolaryngology- } \\
\text { Head \& Neck Surgery }\end{array}$ & wissenschaftliche Fachzeitschrift & USA & 4449 & 4216 & 07.07.2009 \\
\hline @MenieresSociety & Meniere's Society & $\begin{array}{l}\text { Stiftung und } \\
\text { Selbsthilfevereinigung }\end{array}$ & $\begin{array}{l}\text { Vereinigtes } \\
\text { Königreich }\end{array}$ & 3385 & 1770 & 07.02 .2012 \\
\hline @ENT_UK & $\begin{array}{l}\text { The British Association of } \\
\text { Otorhinolaryngology }\end{array}$ & $\begin{array}{l}\text { Fachgesellschaft für Hals-Nasen- } \\
\text { Ohrenheilkunde }\end{array}$ & $\begin{array}{l}\text { Vereinigtes } \\
\text { Königreich }\end{array}$ & 2150 & 2110 & 11.11.2011 \\
\hline @UCLEarlnstitute & $\begin{array}{l}\text { UCL Ear Institute, University } \\
\text { College London (UCL) }\end{array}$ & Institut für Hörforschung & $\begin{array}{l}\text { Vereinigtes } \\
\text { Königreich }\end{array}$ & 2039 & 1457 & 02.05 .2012 \\
\hline @CochlearGlobal & Cochlear Ltd. & Medizintechnikunternehmen & Australien & 1674 & 480 & 25.11 .2014 \\
\hline @OtoRhinoLaryn & $\begin{array}{l}\text { Physicians Employment } \\
\text { (PhysEmp) }\end{array}$ & Stellenmarkt für Ärzte & USA & 1473 & 201 & 20.01 .2010 \\
\hline @OandNonline & Otology \& Neurotology & wissenschaftliche Fachzeitschrift & USA & 1384 & 5196 & 28.10.2011 \\
\hline @evidENT_UCL & evidENT & wissenschaftliche Arbeitsgruppe & $\begin{array}{l}\text { Vereinigtes } \\
\text { Königreich }\end{array}$ & 946 & 346 & 21.02.2014 \\
\hline @EandHonline & Ear and Hearing & wissenschaftliche Fachzeitschrift & USA & 763 & 2801 & 28.10.2011 \\
\hline $\begin{array}{l}\text { @Hearing- } \\
\text { Research }\end{array}$ & $\begin{array}{l}\text { American Hearing Research } \\
\text { Foundation (AHRF) }\end{array}$ & $\begin{array}{l}\text { Stiftung zur Förderung der } \\
\text { Hörforschung }\end{array}$ & USA & 639 & 883 & 04.04 .2012 \\
\hline @HNOPraxisRuhr & HNO Praxis Essen & Praxis & Deutschland & 39 & 120 & 25.09 .2014 \\
\hline @hnoprax & HNO Praxis Wuppertal & Praxis & Deutschland & 41 & 7 & 19.03.2013 \\
\hline @HNOAssistent & $\begin{array}{l}\text { Assistent* innenvertretung } \\
\text { der deutschen Gesellschaft } \\
\text { für HNO }\end{array}$ & $\begin{array}{l}\text { Teil einer Fachgesellschaft für } \\
\text { Hals-Nasen-Ohrenheilkunde }\end{array}$ & Deutschland & 6 & 23 & 22.05 .2018 \\
\hline @DGHNOKHC & $\begin{array}{l}\text { Deutsche Gesellschaft für } \\
\text { Hals-Nasen-Ohren-Heilkunde, } \\
\text { Kopf- und Hals-Chirurgie e.V. }\end{array}$ & $\begin{array}{l}\text { Fachgesellschaft für Hals- } \\
\text { Nasen-Ohrenheilkunde }\end{array}$ & Deutschland & 4 & 26 & 02.05 .2011 \\
\hline @Dr_Reichel & $\begin{array}{l}\text { Dr. Jochen Reichel, } \\
\text { München }\end{array}$ & niedergelassener HNO-Arzt & Deutschland & 4 & 102 & 29.05.2008 \\
\hline @Praxis_HNO & HNO Praxis Ingolstadt & Praxis & Deutschland & 1 & 13 & 26.06.2017 \\
\hline
\end{tabular}

Stand: 09.09.2018

YouTube ist eine der meistfrequentierten Seiten des Internets. Die Möglichkeit, Videos zu verbreiten, bietet besondere Chancen für die Patientenaufklärung [155, 156]. Aufgrund des systemimmanenten Mangels einer zentralen oder systematischen Qualitätssicherungsinstanz enthalten YouTube-Videos zu medizinischen Themen jedoch wahrscheinlich einen sehr hohen Anteil falscher Informationen. Die Verbreitung von anekdotischen oder falschen Informationen ist daher ein großes Risiko dieser Plattform [156]. Gezeigt wurde dies in einer wissenschaftlichen Untersuchung von Videos zu Bewegungsstörungen, die zu $66 \%$ fehlerhafte Einordnungen der darge- stellten Fälle aufwiesen [157]. Hierfür steht eine Lösung mit der nötigen Breitenwirkung auf die unüberschaubare Menge an Material dieser Plattform noch aus. Daneben sollte wissenschaftlich untersucht werden, wie valide Patienteninformationen auf YouTube möglichst optimal verbreitet werden können [156].

YouTube bietet auch Inhalte aus dem Bereich der Hals-Nasen-Ohrenheilkunde, deren Qualität bereits wissenschaftlich evaluiert wurde. Dabei gibt es jedoch noch keinen Konsens im Hinblick auf Beurteilungsinstrumente. Nur eine einzige Studie [158] verwendet eine früher verwendetes validiertes Instrument [159]. Eine weitere Studie ori- 
- Tab. 5 Übersicht zu Analysen von YouTube-Inhalten mit Bezug zur Hals-Nasen-Ohrenheilkunde.

\begin{tabular}{|c|c|c|c|c|c|c|}
\hline Erkrankung/Prozedur & $\begin{array}{l}\text { Zeitpunkt der } \\
\text { Untersuchung }\end{array}$ & Sprache & Suchbegriffe & $\begin{array}{l}\text { Anzahl } \\
\text { Videos }\end{array}$ & $\begin{array}{l}\text { Beurteilungs- } \\
\text { instrument }\end{array}$ & Referenz \\
\hline Pseudokrupp ${ }^{a}$ & 06.10 .2015 & Englisch & $\begin{array}{l}\text { croup + child OR } \\
\text { croup + baby }\end{array}$ & 38 & $\begin{array}{l}\text { Medical Video Rating } \\
\text { System (MVRS) [159] }\end{array}$ & Knight et al. [158] \\
\hline Mundhöhlenkarzinom & $\begin{array}{l}\text { 04. und } \\
05.12 .2015\end{array}$ & Englisch & $\begin{array}{l}\text { mouth cancer } \\
\text { oral cancer }\end{array}$ & 188 & ad hoc entwickelt & Hassona et al. [163] \\
\hline Sjögren-Syndrom & 21.05 .2015 & Englisch & $\begin{array}{l}\text { Sjogren's } \\
\text { syndrome }\end{array}$ & 36 & $\begin{array}{l}\text { angepasst von früheren } \\
\text { Untersuchungen }\end{array}$ & Delli etal. [160] \\
\hline $\begin{array}{l}\text { Adenostonsillektomie, } \\
\text { Paukenröhrchen }\end{array}$ & $\begin{array}{l}\text { 04. und } \\
07.02 .2013\end{array}$ & Englisch & $b$ & 102 & ad hoc entwickelt & Sorensen et al. [162] \\
\hline Rhinosinusitis & $\begin{array}{l}\text { 17. und } \\
18.08 .2012\end{array}$ & Englisch & sinusitis & 100 & ad hoc entwickelt & Biggs et al. [164] \\
\hline $\begin{array}{l}\text { plastische Chirurgie der } \\
\text { Ohrmuschel }\end{array}$ & $\begin{array}{l}\text { nicht } \\
\text { angegeben }\end{array}$ & Englisch & otoplasty & 50 & analog zu[161] & Nissan et al. [121] \\
\hline Facelift & nicht angegeben & Englisch & facelift & 99 & $\begin{array}{l}\text { ad hoc entwickelt, } \\
\text { verwendet auch in [121] }\end{array}$ & Nissan et al. [161] \\
\hline
\end{tabular}

a: Untersucht wurde auch Hypovolämie im Kindesalter. Hier angegeben sind nur die erhobenen Werte in Bezug auf Pseudokrupp; b: Diese Studie verwendete eine Suchanfrage, die für eine möglichst vollständige Abfrage konstruiert wurde, aber wahrscheinlich nicht die Suchgewohnheiten von Patienten oder Laien wiedergibt: „Tonsillectomy“ OR ,adenoidectomy“ OR „tonsil removal“ OR „adenoid removal“ OR „tonsil surgery“ OR „adenoid surgery“ OR „tonsillitis surgery“; „Ear tubes“ OR „ear tube surgery“ OR „ear infection surgery“ OR „myringotomy“ OR „tympanostomy tubes".

entiert sich bei der Beurteilung an früheren Publikationen [160], 2 Publikationen nehmen Bezug aufeinander [121, 161] und ansonsten werden ad hoc-entwickelte Skalen verwendet [162-164]. Die zusammenfassende Beurteilung der Qualität und des Nutzens der Inhalte ist in vielen Fällen allerdings negativ [158, 162, 164]. Besonders Videos, die von einzelnen Nutzern hochgeladen wurden, haben eine geringere Qualität als solche von Angehörigen von Gesundheitsberufen, Institutionen oder professionellen Organisationen [160,163]. Eine Übersicht zu YouTube in der HNO findet sich in $\triangleright$ Tab. $\mathbf{5}$.

Patientenportale sind themenspezifische soziale Netzwerke für das Gesundheitswesen [16]. Die über das Internet vermittelte Interaktion zwischen Patienten wird schon lange als potentiell relevanter Faktor angesehen, wobei die systematische wissenschaftliche Erfassung aufgrund der Vielfalt im Internet schwierig ist $[22,165]$. Allerdings können Patientenportale wie z. B. PatientsLikeMe [166] auch für die Forschung relevant sein, da hierdurch neue Möglichkeiten für die retrospektive Evaluation von Patientendaten entstehen. Dies erfolgte bspw. in einem Fall für den Wirkstoff Lithium, welcher für die Behandlung von Amyotropher Lateralsklerose vorgeschlagen worden war. Ausgangspunkt war eine Studie an 16 Patienten und 28 Kontrollen, die durch die Applikation in einem Zeitraum von 15 Monaten einen signifikant geringeren Krankheitsprogress zeigten [167]. Eine von Patienten angeregte Analyse der Daten des Portals PatientsLikeMe konnte in kurzer Zeit 149 Patienten, die mit Lithium behandelt wurden, sowie 447 Kontrollen identifizieren und analysieren. Dabei wurde kein Effekt von Lithium in einem Zeitraum von bis zu 12 Monaten gefunden [168]. In gleicher Weise konnte an 3342 Fallberichten der off-label Gebrauch von Amitryptilin und Modafinil evaluiert werden [169]. Anwendungen in der Hals-Nasen-Ohrenheilkunde sind bisher nicht bekannt.

\section{5. digitale Gesundheitskompetenz}

\subsection{Kompetenz und Gesundheit}

Bezogen auf den individuellen Patienten dienen Gesundheitsinformationen dazu, die eigenen Fähigkeiten und Fertigkeiten im Umgang mit Gesundheitsfragen zu entwickeln. Hierfür wurde das Konzept der Gesundheitskompetenz (health literacy) eingeführt. Die Übersetzung des Begriffs ist dabei nicht wörtlich erfolgt. „Literacy“ ist im Englischen weiter gefasst als die wörtliche deutsche Übersetzung „Lesefähigkeit“ bzw. „Alphabetisierung“. „Literacy“ beinhaltet neben Lese- und Schreibfähigkeit auch Aspekte der Medienkompetenz. Dies muss beim Verständnis des deutschen Begriffes „Gesundheitskompetenz“ für das englische „health literacy“ berücksichtigt werden [170]. Die American Medical Association definierte noch 1999 Gesundheitskompetenz als die Fähigkeiten, welche nötig sind, um Gesundheitsinformationen zu lesen, zu verstehen und danach als Patient adäquat handeln zu können [171]. Aktuell versteht man unter Gesundheitskompetenz ein weiter reichendes Konzept, das neben den Fähigkeiten, Gesundheitsinformationen zu beschaffen, zu verstehen, zu bewerten und zu befolgen auch die individuellen und sozialen Faktoren beinhaltet, die dies beeinflussen [172, 173].

Diese Fähigkeiten und Faktoren sind eng mit dem Medium verknüpft und unterliegen derzeit einem Wandel, da das Internet gerade zum dominierenden Medium für Kommunikation und Information im Gesundheitswesen wird [70]. In der Folge wurde der Begriff der Gesundheitskompetenz erweitert, um relevante Kompetenzen in der Benutzung von digitalen Medien mit einzubeziehen. In Anlehnung an eHealth findet sich nun der Begriff eHealth literacy (electronic health literacy, etwa digitale Gesundheitskompetenz) [173]. Digitale Ge- 
sundheitskompetenz beinhaltet nach Norman und Skinner 6 Komponenten in 2 Gruppen von Fähigkeiten: Lesen/Rechnen, Informationskompetenz und Medienkompetenz als analytische Fähigkeiten sowie (analoge) Gesundheitskompetenz, EDV-Kenntnisse und naturwissenschaftliche Grundbildung als kontext-spezifische Fähigkeiten [174]. Es ist noch unklar, wie digitale Gesundheitskompetenz am besten erfasst werden kann und welche Konsequenzen sich für die Entwicklung und Implementierung von digitalen Anwendungen im Gesundheitswesen ergeben [175].

Die Gesundheitskompetenz wirkt sich auf den individuellen Gesundheitszustand aus, dieser Zusammenhang wurde in mehreren systematischen Übersichtsarbeiten belegt $[176,177]$. So wurde ein starker Zusammenhang zwischen der Gesundheitskompetenz und der Sterblichkeit von älteren Menschen festgestellt [177]. Menschen mit geringer Gesundheitskompetenz haben ein 1,5- bis 3-fach höheres Risiko für einen ungünstigen Krankheitsverlauf [176]. Weiterhin bestehen Assoziationen mit Krankenhausaufnahmen, Inanspruchnahme von Notfalleinrichtungen, seltenerer Teilnahme an Impfungen und Vorsorgeuntersuchungen wie Mammographie sowie bei älteren Menschen sogar mit einem schlechteren Gesundheitszustand allgemein [177]. Diese Ergebnisse basieren hauptsächlich auf Studien aus den USA, wo es lange keine allgemeine Krankenversicherungspflicht gab und daher Verzerrungen in Bezug auf den sozioökonomischen Status denkbar wären. Es finden sich allerdings auch ähnliche Ergebnisse in einer in Großbritannien über einen Zeitraum von mehr als 5 Jahren durchgeführten Langzeitstudie an $7857 \mathrm{~Pa}-$ tienten, welche 52 Jahre oder älter waren. Aufgrund der ähnlichen Gesundheitssysteme sind diese Daten wahrscheinlich eher mit der Situation in Deutschland vergleichbar. [178].

Digitale Gesundheitskompetenz ist relevant für den Verlauf und die Prognose von Krankheiten. Für Tumorpatienten wurde ein detaillierter konzeptioneller Rahmen vorgeschlagen, wie das Internet durch Inhalte sowie die Vermittlung von Interaktion mit Ärzten und Mitpatienten den Ausgang einer Tumorerkrankung beeinflussen kann [179]. Eine geringe Gesundheitskompetenz scheint auch hier einen negativen Einfluss auf die Fähigkeit zu haben, Gesundheitsinformationen aus dem Internet zu nutzen [180].

\subsection{Faktoren}

Zunächst wäre zu erwarten, dass das Internet als zusätzliches Medium für die Bevölkerung den Zugang zu Gesundheitsinformationen erleichtert $[28,70]$. Da jedoch für die Nutzung des Internets u.a. technische Ressourcen erforderlich sind, ist noch nicht abschließend klar, ob mit dem Internet letztendlich der Erwerb von Gesundheitskompetenz wirklich erleichtert oder sogar erschwert wird [38]. Zudem bedingen verschiedene sozio-ökonomische Merkmale die Ausprägung von Gesundheitskompetenz [28, 173]. Die Rolle des Internets bei der Ermächtigung von Patienten für mehr Verantwortung der eigenen Gesundheit gegenüber kann daher nur im Kontext der sozialen Rahmenbedingungen gesehen werden, in denen das Internet genutzt und damit diese Verantwortung wahrgenommen werden kann [21].

Verschiedene Faktoren wurden beschrieben, die konsistent mit wenig gesundheitsbezogener Internetrecherche und geringer Gesundheitskompetenz assoziiert sind. Dabei scheinen Lebenseinstellung und Bildungshorizont für gesundheitsbezogene Internetrecherche sogar wichtiger zu sein als technische Rahmenbedingungen
[181]. Gerade hohe Bildung wurde als wichtigster Faktor beschrieben, der den Erfolg einer Suche nach validen gesundheitsbezogenen Informationen am besten vorhersagen lässt $[37,182]$, während eine niedrige Bildung mit niedriger Gesundheitskompetenz einher geht [183-186]. Ein ähnlicher Zusammenhang findet sich auf deutscher und europäischer Ebene mit einem niedrigen Sozialstatus [182, 185, 187, 188]. Für einen Einfluss des Alters gibt es widersprüchliche Erkenntnisse. Menschen mit einem Alter von mehr als 65 Jahren suchten am wenigsten nach gesundheitsbezogenen Informationen $[37,182,189]$. Während in vielen Studien ein hohes Alter mit einer niedrigen Gesundheitskompetenz in Verbindung gebracht wird $[184,185]$, fand eine Untersuchung an Mitgliedern der gesetzlichen Krankenversicherung in Deutschland sogar eine steigende Gesundheitskompetenz mit steigendem Alter [190]. Menschen mit Migrationshintergrund in Deutschland nutzen das Internet seltener für die Suche nach Gesundheitsinformationen [188] und ein Migrationshintergrund ist mit geringerer Gesundheitskompetenz assoziiert [185]. Chronisch kranke Patienten nutzen das Internet seltener und können daher nur einen geringen Nutzen daraus ziehen [189]. Europaweit scheinen Menschen mit einem selbstangegebenen schlechten Gesundheitszustand auch eine schlechte Gesundheitskompetenz zu haben [187]. Umgekehrt besteht in Deutschland ein enger Zusammenhang zwischen dem Gesundheitsbewusstsein und der Gesundheitskompetenz [186]. In den USA haben Personen, die ethnischen Minderheiten angehören oder Englisch nicht als Muttersprache aufweisen mit größerer Wahrscheinlichkeit eine indadäquate Gesundheitskompetenz [183]. Schließlich nutzen Frauen das Internet häufiger für die Suche nach Gesundheitsinformationen als Männer [36].

Aus der Kombination dieser Merkmale ergeben sich fachspezifisch besondere Patientengruppen, die entweder insgesamt oder über das Internet nicht ausreichend mit Gesundheitsinformationen versorgt sind. In der Hals-Nasen-Ohrenheilkunde könnten bspw. Tumorpatienten aufgrund eines höheren Alters und niedriger formaler Bildung eine Gruppe mit niedrigem Zugang zum Internet und den damit verbundenen Informationen sein [191].

\subsection{Verteilung}

Die genannten Faktoren führen auch dazu, dass gesundheitsbezogene Internetnutzung und Gesundheitskompetenz in der Bevölkerung insgesamt ungleich verteilt ist. Für Deutschland existieren bevölkerungsbezogene Zahlen aus dem Jahr 2013, nach denen $44 \%$ bis 54\% der Erwachsenen eine problematische oder inadäquate Gesundheitskompetenz aufweisen $[185,186]$. In einer Untersuchung von 2014, die nur Mitglieder der gesetzlichen Krankenversicherung Deutschlands betrachtete, waren es sogar 59,5\% der Befragten [190]. Im europäischen Vergleich wird die Verbreitung von Gesundheitskompetenz in Deutschland dennoch als hoch eingeschätzt, schlechtere Werte als Deutschland erreichen bei acht untersuchten Ländern Österreich, Bulgarien, Griechenland, Polen und Spanien. Der Höchstwert wurde in den Niederlanden gefunden, Deutschland lag noch hinter Irland auf Platz 3 [187]. Fachspezifisch für die HalsNasen-Ohrenheilkunde wurde in den USA an ambulanten Patienten festgestellt, dass $10 \%$ eine inadäquate Gesundheitskompetenz und mehr als ein Viertel ein Teildefizit aufwiesen [183].

Aufgrund dieser Bedeutung von Gesundheitskompetenz werden Strategien evaluiert, welche die Gesundheitskompetenz von Patienten erhöhen oder die Folgen einer geringen Gesundheitskompetenz 
reduzieren sollen. Zum Einsatz kommen dabei gestalterische Maßnahmen und audiovisuellen Medien [192]. In Deutschland wurde mit der „Allianz für Gesundheitskompetenz“ im Jahr 2017 eine Initiative gegründet um die Gesundheitskompetenz in Deutschland zu verbessern [193].

\section{Arzt-Patienten-Verhältnis}

\subsection{Wandel durch das Internet}

Die technischen Neuerungen der Digitalisierung und die damit verbundenen Änderungen der Gesundheitskompetenz bei Patienten führen bereits seit Jahren zu einer grundlegenden Änderung im ArztPatienten-Verhältnis [26,194]. Dies wird dadurch erklärt, dass moderne Gesellschaften stark von Massenmedien geprägt sind und damit medienvermittelte Inhalte häufiger von Patienten in das ArztPatienten-Verhältnis eingebracht werden [21]. Wahrscheinlich werden sich Ärzte daher zukünftig auf ein noch partnerschaftlicheres Verhältnis zwischen Arzt und Patient einstellen müssen [194, 195]. Die Situation, dass Informationen über medizinische Sachverhalte einen Patienten unabhängig vom behandelnden Arzt beeinflussen können, ist jedoch nicht neu und es gibt frühere Beispiele bei technischen Neuerungen der Medien in nahezu jeder medizinischen Epoche [196]. Vielleicht sind die Auswirkungen des Internets aber deshalb schwieriger zu bewältigen, weil es die erste Innovation im Gesundheitswesen ist, die nicht vollständig in der Kontrolle des Arztes, sondern mindestens ebenso in der des Patienten ist [197].

Während Patienten das Internet in zunehmendem Maße gesundheitsbezogen nutzen, sind viele Ärzte noch skeptisch [24]. Problematisch für das Arzt-Patienten Verhältnis ist die Tatsache, dass die ärztliche Autorität zunehmend angezweifelt wird. Daneben können aus dem Internet entstandenen Patientenwünsche kontraproduktiv für die Gesundheit sein. Weiterhin werden lange Diskussionen mit Patienten von Ärzten als zeitaufwendig wahrgenommen [179]. Eine Herausforderung der ärztlichen Kompetenz kann bei bestimmten Ärzten zu sehr negativen Reaktionen führen und damit das Arzt-Patienten-Verhältnis deutlich belasten [198]. Das Phänomen des premature consent (sinngemäß: unreife Entscheidung) bezeichnet die Situation, dass ein Patient mit einer bereits vorgefassten Meinung erstmalig einen Arzt konsultiert und aufgrund dieser nicht dem ausdrücklichen Rat des Arztes folgt. Dies hat durch die Verfügbarkeit von Gesundheitsinformationen im Internet wahrscheinlich zugenommen und wird auch als „Dr. Google“ bezeichnet. Scheinbar sind viele Ärzte geneigt, Wünschen von Patienten nachzugeben, die diese aus Internetrecherchen erlangt haben [198]. Aus ethischer Sicht besteht jedoch in einer solchen Situation trotz des gebotenen Respekts vor der Patientenautonomie keine Verpflichtung für den Arzt, einem als unsinnig angesehenen Patientenwunsch zu folgen [199]. Somit ist letztlich die Qualität von Gesundheitsinformationen ausschlaggebend für das Arzt-Patienten-Verhältnis: falsche oder irrelevante Informationen des Patienten können zu einem schlechten Behandlungsergebnis führen [198].

\subsection{Chancen für Verbesserung}

Da das Internet sehr niedrigschwellig und mit hoher Geschwindigkeit einen Informationsaustausch ermöglicht, reduziert es scheinbar die Abhängigkeit des Patienten von Ärzten und ermöglicht eine neue
Form der Eigenständigkeit [24]. Moderne, gut ausgebildete und informierte Patienten wünschen sich dementsprechend bei der Inanspruchnahme von Gesundheitsleistungen in der Regel Zweckmäßigkeit, Kontrolle und Auswahl verschiedener Optionen [195]. Noch ist jedoch nicht absehbar, wie diese gegenwärtige Betonung der Eigenverantwortlichkeit für die Gesundheit letztlich die Rolle des Patienten verändert, weil es wahrscheinlich eine Grenze gibt, bis zu der die Verantwortung für die eigene Gesundheit durch einen Laien ohne professionelle Akteure und den soziokulturellen Kontext sinnvoll übernommen werden kann [21].

Der verbesserte Zugang zu Informationen über das Internet führt dazu, dass Patienten einen größeren Anteil an Entscheidungen in Bezug auf die eigene Gesundheit einfordern [200]. Patienten berichten aber auch von Schwierigkeiten im Umgang mit gesundheitsbezogenen Informationen aus dem Internet. Eine zu große Vielfalt an Informationen, widersprüchliche Erkenntnisse und zu komplexe Informationen werden dabei genannt [201,202] und können letztendlich selbstständige Entscheidungen aufgrund der aus dem Internet gewonnenen Informationen für den Patienten allein unmöglich machen [202]. Es gibt einen Zusammenhang zwischen übermäßiger Nutzung des Internets und der gesundheitsbezogenen Beunruhigung, die aus der Konsultation des Internets in Gesundheitsfragen resultiert [203]. Vielleicht ziehen viele Patienten deswegen das persönliche Gespräch mit dem behandelnden Arzt vor und nutzen Internetseiten als sekundäre Quellen [204]. Ärzte sind hier gefordert, Informationen zu gewichten und zu verifizieren. Dementsprechend bleibt Untersuchungen zufolge das Vertrauen in Ärzte trotz der fortschreitenden Digitalisierung bisher über Jahre hinweg auf hohem Niveau [205]. Das ist positiv zu bewerten, da ein erfolgreiches Ergebnis des Zusammenwirkens von Arzt und Patient Vertrauen im Binnenverhältnis erfordert [206]. Ein weiterer wesentlicher Aspekt für Patienten ist wahrscheinlich der über das Internet vermittelte Kontakt zu anderen Patienten. Dieser Effekt ist allerdings aus methodischen Gründen noch schwer zu quantifizieren [165].

Ärzte sollten sich aktiv um einen positiven Nutzen des Internets für das Arzt-Patienten-Verhältnis bemühen [207]. Beispielsweise könnten Ärzte die Frage nach gesundheitsbezogenen Informationen aus dem Internet in das Anamnesegespräch mit aufnehmen. Patienten bringen von sich aus Vorwissen aus dem Internet wohl nur indirekt in ein Gespräch mit einem Arzt ein [201]. Ärzte scheinen bisher auch die Gesundheitskompetenz von Patienten systematisch zu überschätzen [184], möglicherweise weil das Arzt-Patienten-Verhältnis seit jeher auch von einer Kluft zwischen Lesekompetenz und Hintergrundwissen des sehr gut ausgebildeten und informierten Arztes und dem durchschnittlichen Patienten geprägt ist [67].

In der Mehrzahl der bisherigen Untersuchungen wurde eine positive Einstellung von Ärzten gegenüber Gesundheitsinformationen im Internet dargestellt [179]. Positiv für Ärzte könnte sein, dass Patienten, die sich im Internet informiert haben, mehr Vorwissen über Gesundheitsthemen haben [198, 200-202]. Voraussetzung für eine Verbesserung des Verhältnisses zwischen Arzt und Patient durch die Digitalisierung bleibt aber eine kritische Betrachtung der Nachteile und eine Realisierung des für die Patienten möglichen Gewinns an Eigenständigkeit [29]. Vielfach wird es unabhängig vom Medium als Pflicht des Arztes gesehen, dafür Sorge zu tragen, dass Patienten angemessen informiert sind [146]. Um im Interesse des Patienten handeln zu können, hat der Arzt auch die ethische Verpflichtung, diesen 
a

Urheber

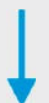

Gesundheitsinformationen (klassische Medien)

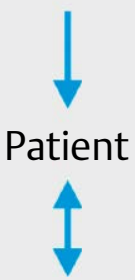

Arzt
Urheber
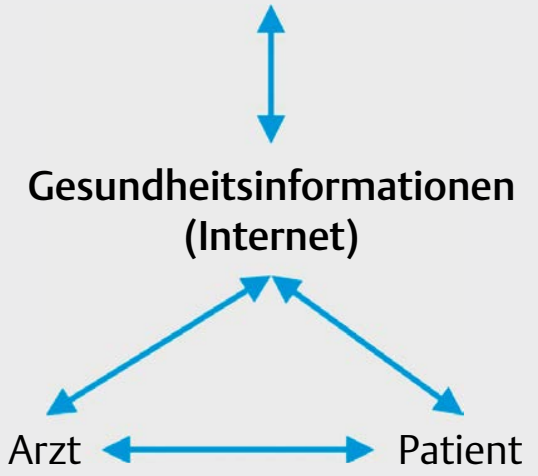

- Abb. 2 Vereinfachtes Schema zum Arzt-Patienten-Verhältnis. a Situation ohne Internet, b Situation mit Internet. Das Internet bewirkt v. a. eine Zunahme der möglichen Interaktionen.

vor Gefahren zu schützen, die durch mangelnde oder falsche Informationen auf Seiten des Patienten zustande kommen [206]. Das aktuelle Modell zur Entscheidungsfindung in Gesundheitsfragen beinhaltet eine Diskussion der Vorteile und Nachteile verschiedener Optionen und eine Berücksichtigung der persönlichen Werte des individuellen Patienten (shared decision making) [200]. Letztlich ist es damit Aufgabe des ärztlichen Berufsstandes, sicherzustellen, dass die digitalisierte Medizin das Gesundheitswesen verbessert [26].

Auch in der HNO berichten Patienten nicht unbedingt von sich aus von Vorwissen aus dem Internet. Dennoch kann dadurch die Einstellung zu Erkrankung, Arzt oder Therapieverfahren beeinflusst sein $[45,48]$. Anhand der Rhinoplastik konnte dargestellt werden, dass bei plastisch-ästhetischen Eingriffen das Internet als Marketinginstrument eine besonders wichtige Rolle zu spielen scheint [208]. Obwohl aber gerade bei ästhetischen Indikationen der Nutzungsgrad des Internets sehr hoch ist, scheint an diesem Beispiel besonders deutlich zu werden, dass der direkte Kontakt zum Arzt unersetzlich ist, da Informationen, die vom behandelnden Arzt kommen, in der Regel genauer und mehr auf die Person zugeschnitten sind [209]. Insgesamt führt die Verfügbarkeit des Internets zu erhöhter Interaktion von Arzt, Patient und den verfügbaren Gesundheitsinformationen. Ein Schema dazu findet sich in $>$ Abb. 2.

\section{Schlussfolgerungen, Ausblick und wei- terführende Ansätze}

Das Internet stellt eine technische Neuerung dar, die als Medium und Kommunikationsmittel alle Lebensbereiche des Menschen durchdringt. Betroffen ist auch das Gesundheitswesen, in dem sich die Verfügbarkeit von Informationen und die Schnelligkeit von Kommunikation auf die Gesundheitskompetenz der Patienten sowie das Verhältnis zwischen Arzt und Patienten auswirken. Mittelbar gibt es dadurch Auswirkungen auf den Behandlungserfolg von Erkrankungen.
- Tab. 6 mögliche Maßnahmen in Bezug auf digitale Gesundheitskompetenz

Öffentlichkeitsarbeit

- koordinierte Erstellung vertrauenswürdiger Gesundheitsinformationen

- Etablierung einer dauerhaften Präsenz in den sozialen Medien gegenüber Laien, Patienten und Kollegen

wissenschaftliches Arbeitsprogramm

- Zugänglichkeit und Qualität deutschsprachiger Gesundheitsinformationen zu Krankheitsbildern und Prozeduren

- Verteilung, Barrieren und Effekt der fachbezogenen Gesundheitskompetenz

- Erarbeitung und Evaluation von Interventionen zur Verbesserung der fachbezogenen Gesundheitskompetenz

Die Hals-Nasen-Ohrenheilkunde ist von diesem Prozess naturgemäß mit betroffen. Detaillierte wissenschaftliche Erkenntnisse liegen im Fachgebiet bisher zur Internetnutzung von Patienten sowie zu englischsprachigen Gesundheitsinformationen vor. Forschungsbedarf besteht in Bezug auf die Verfügbarkeit und Qualität von deutschsprachigen Gesundheitsinformationen im Internet, zum Stellenwert des Internets sowie zu Hindernissen beim Zugang für Patienten der Hals-Nasen-Ohrenheilkunde in den Rahmenbedingungen des deutschen Gesundheitssystems.

Jedes medizinische Fach ist gefordert, den durch die Digitalisierung bedingten Wandel in der Gesundheitskompetenz der behandelten Patienten aktiv mit zu gestalten. Neben der praktischen Verbesserung der fachbezogenen digitalen Gesundheitskompetenz durch gezielte Öffentlichkeitsarbeit sollten die fachspezifischen Auswirkungen der Digitalisierung wissenschaftlich evaluiert werden. Eine Übersicht über mögliche Maßnahmen findet sich in > Tab. 6.

\section{Interessenkonflikt}

Die Autoren geben an, dass kein Interessenkonflikt besteht. 
[1] Liberati A, Altman DG, Tetzlaff J, Mulrow C, Gøtzsche PC, loannidis JPA, Clarke M, Devereaux PJ, Kleijnen J, Moher D. The PRISMA statement for reporting systematic reviews and meta-analyses of studies that evaluate healthcare interventions: explanation and elaboration. BMJ 2009; 339: b2700 Im Internet: http://www.ncbi. nlm.nih.gov/pubmed/19622552

[2] Moher D, Liberati A, Tetzlaff ], Altman DG.PRISMA Group. Preferred reporting items for systematic reviews and meta-analyses: the PRISMA statement. BMJ 2009; 339: b2535 Im Internet: http://www. ncbi.nlm.nih.gov/pubmed/19622551

[3] Leiner BM, Cerf VG, Clark DD, Kahn RE, Kleinrock L, Lynch DC, Postel J, Roberts LG, Wolff S. A brief history of the internet. ACM SIGCOMM. Comput Commun Rev.2009: 39: 22 Im Internet http://portal.acm. org/citation.cfm?doid $=1629607.1629613$

[4] Höflich JR. Internet und E-Mail - neue Wege schriftlicher Kommunikation? In: Der Mensch und seine Medien.Wiesbaden: Springer Fachmedien Wiesbaden; 2016: 115-131 Im Internet: http://link. springer.com/10.1007/978-3-531-18683-2_7

[5] Rosenzweig R. Wizards, Bureaucrats, Warriors, and Hackers: Writing the History of the Internet. Am Hist Rev 1998; 103: 1530 Im Internet http://www.jstor.org.prox.lib.ncsu.edu/sici?sici $=0002$ -

8762(1998)103:5\%3C1530:WBWAHW\%3E2.0.CO;2-U\&origin = serialsolutions\&

[6] Cerf V, Kahn R. A Protocol for Packet Network Intercommunication. IEEE Trans Commun 1974; 22: 637-648 Im Internet http:// ieeexplore.ieee.org/document/1092259/

[7] Berners-Lee T, Cailliau R, Groff J, Pollermann B. World-Wide Web: The Information Universe. Internet Res 1992; 2: 52-58 Im Internet: http://www.emeraldinsight.com/doi/10.1108/eb047254

[8] Ben Mokhtar S, Boutet A, Felber P, Pasin M, Pires R, Schiavoni V.X-search. In: Proceedings of the 18th ACM/IFIP/USENIX Middleware Conference on - Middleware '17. New York, New York, USA: ACM Press, 2017: 198-208 Im Internet: http://dl.acm.org/citation. cfm?doid $=3135974.3135987$

[9] Kobayashi M, Takeda K. Information retrieval on the web. ACM Comput Surv 2000; 32: 144-173 Im Internet http://portal.acm.org/ citation.cfm?doid $=358923.358934$

[10] Brin S, Page L. The anatomy of a large-scale hypertextual Web search engine. Comput Networks ISDN Syst 1998; 30: 107-117 Im Internet https://www.sciencedirect.com/science/article/pii/ S016975529800110X?via \%3Dihub

[11] O’Reilly T.What Is Web 2.0 - Design Patterns and Business Models for the Next Generation of Software. 2005; Im Internet: https://www. oreilly.com/pub/a/web2/archive/what-is-web-20.html

[12] DiNucci D. Fragmented Future. Print 1999; 32: 221-222 Im Internet http://darcyd.com/fragmented_future.pdf

[13] Kamel Boulos MN, Wheeler S. The emerging Web 2.0 social software: an enabling suite of sociable technologies in health and health care education 1. Heal Inf Libr J.2007: 24: 2-23 Im Internet: http://doi. wiley.com/10.1111/j.1471-1842.2007.00701.x

[14] Zhao WX, Jiang J, Weng J, He J, Lim E, Yan H, Li X. Comparing Twitter and Traditional Media using Topic Models. Proc 33 ${ }^{\text {rd }}$ Eur Conf Adv Inf Retr 2011; 338-349

[15] Stein K, Hess C. Viele Autoren, gute Autoren? Eine Untersuchung ausgezeichneter Artikel in der deutschen Wikipedia. In: Web $2.0-$ Eine empirische Bestandsaufnahme.Wiesbaden: Vieweg + Teubner; 107-129 Im Internet: http://www.springerlink.com/index/10.1007/978-3-8348-9498-4_6

[16] Grajales FJ, Sheps S, Ho K, Novak-Lauscher H, Eysenbach G. Social media: a review and tutorial of applications in medicine and health care. J Med Internet Res 2014; 16: e13 Im Internet: http://www.jmir. org/2014/2/e13/
[17] Giles J. Internet encyclopaedias go head to head. Nature 2005; 438 : 900-901 Im Internet http://www.nature.com/articles/438900a

[18] Kong Q, Rizoiu M-A, Wu S, Xie L.Will This Video Go Viral? Explaining and Predicting the Popularity of Youtube Videos. In: Companion of the The Web Conference 2018 on The Web Conference 2018 WWW '18. New York, New York, USA: ACM Press, 2018: 175-178 Im Internet http://dl.acm.org/citation.cfm?doid = 3184558.3186972

[19] Goes PB, Lin M, Au Yeung C. "Popularity Effect" in User-Generated Content: Evidence from Online Product Reviews. Inf Syst Res 2014; 25: 222-238 Im Internet: http://pubsonline.informs.org/doi/ abs/10.1287/isre.2013.0512

[20] Meng M, Agarwal R. Through a glass darkly: Information technology design, identity verification, and knowledge contribution in online communities. Inf Syst Res 2007; 18: 42-67 Im Internet: http:// pubsonline.informs.org/doi/abs/10.1287/isre.1070.0113

[21] Hodgetts D, Bolam B, Stephens C. Mediation and the Construction of Contemporary Understandings of Health and Lifestyle. J Health Psychol 2005; 10: 123-136 Im Internet: http://journals.sagepub. com/doi/10.1177/1359105305048559

[22] Ziebland S, Wyke S. Health and illness in a connected world: how might sharing experiences on the internet affect people's health? Milbank Q 2012; 90: 219-249 Im Internet: http://doi.wiley. com/10.1111/j.1468-0009.2012.00662.x

[23] O'Malley AS. Tapping the Unmet Potential of Health Information Technology. N Engl J Med 2011; 364: 1090-1091 Im Internet: http:// www.nejm.org/doi/abs/10.1056/NEJMp1011227

[24] Anderson JG, Rainey MR, Eysenbach G. The impact of CyberHealthcare on the physician-patient relationship. J Med Syst 2003; 27 : 67-84 Im Internet http://www.ncbi.nlm.nih.gov/pubmed/12617199

[25] Prestin A, Vieux SN, Chou W-YS. Is Online Health Activity Alive and Well or Flatlining? Findings From 10 Years of the Health Information National Trends Survey. J Health Commun 2015; 20: 790-798 Im Internet http://www.ncbi.nlm.nih.gov/pubmed/26042588

[26] Stone JH. Communication between physicians and patients in the era of E-medicine. N Engl J Med 2007; 356: 2451-2454 Im Internet: http://www.nejm.org/doi/abs/10.1056/NEJMp068198

[27] Eysenbach G. What is e-health? J Med Internet Res 2001; 3: e20 Im Internet: http://www.jmir.org/2001/2/e20/

[28] Eysenbach G, Jadad AR. Evidence-based patient choice and consumer health informatics in the Internet age. J Med Internet Res 2001; 3: E19 Im Internet http://www.jmir.org/2001/2/e19/

[29] Capurro R. Skepsis gegenüber Hypes. Dtsch Arztebl 2018; 115: A 1425-A 1428

[30] Al-Ubaydli M. Using Search Engines to Find Online Medical Information. PLoS Med 2005; 2: e228 Im Internet: http://dx.plos. org/10.1371/journal.pmed.0020228

[31] Schoenherr GP, White RW.Interactions between health searchers and search engines. In: Proceedings of the $37^{\text {th }}$ international ACM SIGIR conference on Research \& development in information retrieval - SIGIR '14. New York, New York, USA: ACM Press, 2014: 143-152 Im Internet http://arxiv.org/abs/1712.03622

[32] Wong MKY, Sivasegaran D, Choo CSC, Nah SA. Parental Internet Use and Health Information Seeking Behavior Comparing Elective and Emergency Pediatric Surgical Situations. Eur J Pediatr Surg 2018; 28 : 89-95

[33] Dumitru RC, Bürkle T, Potapov S, Lausen B, Wiese B, Prokosch H. Use and perception of Internet for health related purposes in Germany: results of a national survey. Int J Public Health 2007; 52: 275-285 Im Internet: http://link.springer.com/10.1007/s00038-007-6067-0

[34] Andreassen HK, Bujnowska-Fedak MM, Chronaki CE, Dumitru RC, Pudule I, Santana S, Voss H, Wynn R. European citizens' use of E-health services: A study of seven countries. BMC Public Health 2007; 7: 53 Im Internet: http://bmcpublichealth.biomedcentral.com/ articles/10.1186/1471-2458-7-53 
[35] Kuehn BM. More than one-third of us individuals use the internet to self-diagnose. JAMA - J Am Med Assoc 2013; 309: 756-757

[36] Kummervold PE, Chronaki CE, Lausen B, Prokosch H-U, Rasmussen J, Santana S, Staniszewski A, Wangberg SC. eHealth Trends in Europe 2005-2007: A Population-Based Survey. J Med Internet Res 2008; 10 : e42 Im Internet: http://bmcpublichealth.biomedcentral.com/ articles/10.1186/1471-2458-7-53

[37] Roehr B. Trend for US patients to seek health information from media and internet is stalling. BMJ 2011; 343: d7738 Im Internet: http:// www.bmj.com/cgi/doi/10.1136/bmj.d7738

[38] Jacobs W, Amuta AO, Jeon KC. Health information seeking in the digital age: An analysis of health information seeking behavior among US adults. Cogent Soc Sci 2017; 3: Im Internet: https://www. cogentoa.com/article/10.1080/23311886.2017.1302785

[39] Nelson DE, Kreps GL, Hesse BW, Croyle RT, Willis G, Arora NK, Rimer BK, Viswanath KV, Weinstein N, Alden S. The Health Information National Trends Survey (HINTS): development, design, and dissemination. J Health Commun 2004; 9: 443-460discussion 81-4 Im Internet: http://www.tandfonline.com/doi/ abs/10.1080/10810730490504233

[40] O'Connor JB. Use of the Web for Medical Information by a Gastroenterology Clinic Population. JAMA 2000; 284: 1962 Im Internet: http:// jama.jamanetwork.com/article.aspx?doi $=10.1001 /$ jama.284.15.1962

[41] Rokade A, Kapoor PKD, Rao S, Rokade V, Reddy KTV, Kumar BN. Has the internet overtaken other traditional sources of health information? Questionnaire survey of patients attending ENT outpatient clinics. Clin Otolaryngol Allied Sci 2002; 27: 526-528 Im Internet http://www.ncbi.nlm.nih.gov/pubmed/12472525

[42] Pagedar NA, Schularick NM, Lee PC, Karnell LH. Health-Related Internet Use Among Otolaryngology Patients. Ann Otol Rhinol Laryngol 2018; 127: 551-557 Im Internet: http://journals.sagepub. com/doi/10.1177/0003489418779414

[43] Trotter MI, Morgan DW. Patients' use of the Internet for health related matters: a study of Internet usage in 2000 and 2006 . Health Informatics J 2008; 14: 175-181 Im Internet: http://journals. sagepub.com/doi/10.1177/1081180X08092828

[44] Gurr A, Schwaab M, Hansen S, Noack V, Dazert S. Informationsverhalten von HNO-Patienten im Internet. HNO 2009; 57: 473-479 Im Internet: http://link.springer.com/10.1007/s00106-009-1897-0

[45] Nogueira JF, Rodrigo Hermann D, Solferini Silva ML, Pires Santos F, Nagata Pignatari SS, Cassol Stamm A. Is the information available on the Web influencing the way parents see ENT surgical procedures? Braz J Otorhinolaryngol 2009; 75: 517-523 Im Internet http://www. jmir.org/2001/2/e20/

[46] Shaw B, Farboud A, Trinidade A, Kothari P. Internet and e-mail use in ENT: a survey of patient usage and satisfaction. Eur Arch Oto-RhinoLaryngology 2012; 269: 1051-1054 Im Internet http://link.springer. com/10.1007/s00405-011-1773-x

[47] Boston M, Ruwe E, Duggins A, Willging JP. Internet Use by Parents of Children Undergoing Outpatient Otolaryngology Procedures. Arch Otolaryngol Neck Surg 2005; 131: 719 Im Internet http://www.ncbi. nlm.nih.gov/pubmed/16103305

[48] Glynn RW, O’Duffy F, O'Dwyer TP, Colreavy MP, Rowley HM, O'Duffy F, O'Dwyer TP, Colreavy MP, Rowley HM. Patterns of Internet and smartphone use by parents of children attending a pediatric otolaryngology service. Int J Pediatr Otorhinolaryngol 2013; 77: 699-702 Im Internet: http://dx.doi.org/10.1016/j.ijporl.2013.01.021

[49] Thorén ES, Öberg M, Wänström G, Andersson G, Lunner T. Internet access and use in adults with hearing loss. J Med Internet Res 2013; 15: e91 Im Internet: http://www.jmir.org/2013/5/e91/

[50] Tassone P, Georgalas C, Patel NN, Appleby E, Kotecha B. Do otolaryngology out-patients use the internet prior to attending their appointment? J Laryngol Otol 2004; 118: 34-38 Im Internet http:// www.journals.cambridge.org/abstract_S002221510400009X
[51] Tuffrey C, Finlay F. Use of the internet by parents of paediatric outpatients. Arch Dis Child 2002; 87: 534-536 Im Internet http:// www.ncbi.nlm.nih.gov/pubmed/12456558

[52] Eysenbach G, Köhler C. How do consumers search for and appraise health information on the world wide web? Qualitative study using focus groups, usability tests, and in-depth interviews. BMJ 2002; 324: 573-577 Im Internet http://www.ncbi.nlm.nih.gov/pubmed/11884321

[53] Morahan-Martin JM. How internet users find, evaluate, and use online health information: a cross-cultural review. Cyberpsychol Behav 2004; 7: 497-510 Im Internet: http://www.liebertonline.com/doi/ abs/10.1089/cpb.2004.7.497

[54] Toms EG, Latter C. How consumers search for health information. Health Informatics J 2007; 13: 223-235

[55] Boyer C, Gaudinat A, Hanbury A, Appel RD, Ball M]. Accessing Reliable Health Information on the Web : A Review of the HON Approach. 2017; 1004-1008

[56] Pletneva N, Ruiz de Castaneda R, Baroz F, Boyer C. General vs health specialized search engine: a blind comparative evaluation of top search results. Stud Health Technol Inform 2014; 205: 201-205 Im Internet http://www.ncbi.nlm.nih.gov/pubmed/25160174

[57] Muse K, McManus F, Leung C, Meghreblian B, Williams JMG. Cyberchondriasis: Fact or fiction? A preliminary examination of the relationship between health anxiety and searching for health information on the Internet. J Anxiety Disord 2012; 26: 189-196 Im Internet: doi:http://dx.doi.org/10.1016/j.janxdis.2011.11.005

[58] Bessiere K, Pressman S, Kiesler S, Kraut R. Effects of Internet use on health and depression: A longitudinal study. J Med Internet Res 2010; 12: 6-7

[59] White RW, Horvitz E. Cyberchondria: studies of the escalation of medical concerns in web search. ACM Trans. Inf Syst 2009; 27: 1-37 Im Internet http://dl.acm.org/citation.cfm?id = 1629096.1629101

[60] Mathieu E. The Internet and Medical Decision Making: Can It Replace the Role of Health Care Providers? Med Decis Mak 2010; 30: 14-16 Im Internet: http://journals.sagepub.com/ doi/10.1177/0272989X10381228

[61] Crocco AG, Villasis-Keever M, Jadad AR. Analysis of cases of harm associated with use of health information on the internet. JAMA 2002; 287: 2869-2871 Im Internet http://www.ncbi.nlm.nih.gov/ pubmed/12038937

[62] Farmer SE], Bernardotto M, Singh V. How good is Internet self-diagnosis of ENT symptoms using Boots WebMD symptom checker? Clin Otolaryngol 2011; 36: 517-518 Im Internet: http://doi.wiley. com/10.1111/j.1749-4486.2011.02375.x

[63] Silberg WM, Lundberg GD, Musacchio RA. Assessing, controlling, and assuring the quality of medical information on the Internet: Caveant lector et viewor--Let the reader and viewer beware. JAMA 1997; 277: 1244-1245 Im Internet http://www.ncbi.nlm.nih.gov/pub$\operatorname{med} / 9103351$

[64] Purcell GP, Wilson P, Delamothe T. The quality of health information on the internet. BMJ 2002; 324: 557-558 Im Internet http://www. bmj.com.kuleuven.ezproxy.kuleuven.be/content/bmj/324/7337/557. full.pdf

[65] Kim H, Xie B. Health literacy in the eHealth era: A systematic review of the literature. Patient Educ Couns 2017; 100: 1073-1082 Im Internet: http://dx.doi.org/10.1016/j.pec.2017.01.015

[66] Eysenbach G, Powell J, Kuss O, Sa E-R. Empirical Studies Assessing the Quality of Health Information for Consumers A Systematic Review. JAMA 2002; 287: 2691-2700 Im Internet http://www.ncbi.nlm.nih. gov/pubmed/12020305

[67] Sun $\mathrm{GH}$. The digital divide in Internet-based patient education materials. Otolaryngol Head Neck Surg 2012; 147: 855-857 Im Internet: http://journals.sagepub.com/ doi $/ 10.1177 / 0194599812456153$ 
[68] Volsky PG, Baldassari CM, Mushti S, Derkay CS. Quality of Internet information in pediatric otolaryngology: A comparison of three most referenced websites. Int J Pediatr Otorhinolaryngol 2012; 76: 1312-1316 Im Internet: http://dx.doi.org/10.1016/j.ijporl.2012.05.026

[69] Koh HK, Brach C, Harris LM, Parchman ML. A proposed „health literate care model" would constitute a systems approach to improving patients' engagement in care. Health Aff (Millwood) 2013; 32: 357-367 Im Internet: http://www.healthaffairs.org/doi/10.1377/ hlthaff. 2012.1205

[70] McCray AT. Promoting Health Literacy. J Am Med Informatics Assoc 2004; 12: 152-163 Im Internet http://171.67.114.118/content/12/2/152.abstract

[71] Walsh TM, Volsko TA. Readability assessment of internet-based consumer health information. Respir Care 2008; 53: 1310-1315 Im Internet http://www.ncbi.nlm.nih.gov/entrez/query.fcgi?cmd = Retrie ve \&db $=$ PubMed \&dopt $=$ Citation\&list_uids $=18811992 \% 5$ Cnhttp: $/ /$ rc.rcjournal.com/content/53/10/1310.full.pdf

[72] Ley P, Florio T. The use of readability formulas in health care. Psychol Heal Med 1996; 1: 7-28

[73] Friedman DB, Hoffman-Goetz L. A systematic review of readability and comprehension instruments used for print and web-based cancer information. Heal Educ Behav 2006; 33: 352-373

[74] Wang L-W, Miller M], Schmitt MR, Wen FK. Assessing readability formula differences with written health information materials: application, results, and recommendations. Res Social Adm Pharm 2013; 9: 503-516 Im Internet: http://dx.doi.org/10.1016/j.sapharm. 2012.05.009

[75] Flesch R. A new readability yardstick. J Appl Psychol 1948; 32: 221-233 Im Internet http://doi.apa.org/getdoi.cfm?doi = 10.1037/ h0057532

[76] Amstad T. Wie verständlich sind unsere Zeitungen? 1978

[77] Kincaid JP, Fishburne J, Robert PR, Richard LC, Brad S.Derivation of New Readability Formulas (Automated Readability Index, Fog Count and Flesch Reading Ease Formula) for Navy Enlisted Personnel. 1975 Im Internet: http://www.dtic.mil/docs/citations/ADA006655

[78] Gunning R. The technique of clear writing. New York, New York, USA: McGraw-Hill; 1952

[79] McLaughlin GH. SMOG grading: A new readability formula. J Read 1969; 12: 639-646 Im Internet http://www.jstor.org/stable/4001 1226

[80] Wild J, Pissarek M. Regensburger Analysetool für Texte. 2016; 12

[81] Lenhard W, Lenhard A. Berechnung des Lesbarkeitsindex LIX nach Björnson. Bibergau 2014 Im Internet https://www.psychometrica. de/lix.html

[82] Charnock D, Shepperd S, Needham G, Gann R. DISCERN: an instrument for judging the quality of written consumer health information on treatment choices. J Epidemiol Community Heal 1999; 53: 105-111 Im Internet: http://jech.bmj.com/cgi/ doi/10.1136/jech.53.2.105

[83] Allam A, Schulz PJ, Krauthammer M. Toward automated assessment of health Web page quality using the DISCERN instrument. J Am Med Informatics Assoc 2016; 24: ocw140 Im Internet https://academic. oup.com/jamia/article-lookup/doi/10.1093/jamia/ocw140

[84] Charnock D. The discern handbook.Oxford: Radcliffe Medical Press Ltd; 1998Im Internet: http://www.discern-genetics.org/discern. pdf\%5Cnhttp://www.webcitation.org/6MhdgErhv

[85] Rees CE, Ford JE, Sheard CE. Evaluating the reliability of DISCERN: a tool for assessing the quality of written patient information on treatment choices. Patient Educ Couns 2002; 47: 273-275 Im Internet http://www.ncbi.nlm.nih.gov/pubmed/12088606
[86] Charnock D, Shepperd S. Learning to DISCERN online: applying an appraisal tool to health websites in a workshop setting. Health Educ Res 2004; 19: 440-446 Im Internet https://academic.oup.com/her/ article-lookup/doi/10.1093/her/cyg046

[87] Boyer C, Selby M, Appel RD, Scherrer JR, Appel RD. The Health On the Net Code of Conduct for medical and health Websites. Comput Biol Med 1998; 28: 603-610 Im Internet http://www.ncbi.nlm.nih.gov/ pubmed/9861515

[88] Boyer C, Appel RD, Ball MJ, Van Bemmel JH, Bergmans JP, Carpentier M, Hochstrasser D, Lindberg D, Miller R, Peterschmitt JC, Safran C, Thonnet M, Geissbühler A. Health on the net's 20 years of transparent \& reliable health information. Stud Health Technol Inform 2017; 228: 700-704

[89] Boyer C, Dolamic L, Grabar N. Automated Detection of Health Websites' HONcode Conformity: Can N-gram Tokenization Replace Stemming? Stud Health Technol Inform 2015; 216: 1064 Im Internet http://www.ncbi.nlm.nih.gov/pubmed/26262363

[90] Boyer C, Dolamic L. Automated Detection of HONcode Website Conformity Compared to Manual Detection: An Evaluation. J Med Internet Res 2015; 17: e135 Im Internet: http://www.jmir.org/2015/6/e135/

[91] Miller N, Lacroix EM, Backus JE. MEDLINEplus: building and maintaining the National Library of Medicine's consumer health Web service. Bull Med Libr Assoc 2000; 88: 11-17 Im Internet http://www. ncbi.nlm.nih.gov/pubmed/10658959

[92] Meyer MF, Bacher R, Roth KS, Beutner D, Luers JC. Systematische Analyse der Lesbarkeit von Patienteninformationstexten auf Internetseiten deutscher nichtuniversitärer HNO-Kliniken. HNO 2014; 62: 186-195

[93] Luers J-C, Gostian A-O, Roth KS, Beutner D. Lesbarkeit von medizinischen Texten im Internetangebot deutscher HNO-Universitätskliniken. HNO.2013: 61: 648-654 Im Internet: http://link.springer. com/10.1007/s00106-013-2674-7

[94] Svider PF, Agarwal N, Choudhry OJ, Hajart AF, Baredes S, Liu JK, Eloy JA. Readability assessment of online patient education materials from academic otolaryngology-head and neck surgery departments. Am J Otolaryngol - Head Neck Med Surg 2013; 34: 31-35 Im Internet: doi:http://dx.doi.org/10.1016/j.amjoto.2012.08.001

[95] Eloy JA, Li S, Kasabwala K, Agarwa N, Hansberry DR, Baredes S, Setzen M. Readability assessment of patient education materials on major otolaryngology association websites. Otolaryngol - Head Neck Surg (United States) 2012; 147: 848-854

[96] Kasabwala K, Agarwal N, Hansberry DR, Baredes S, Eloy JA. Readability Assessment of Patient Education Materials from the American Academy of Otolaryngology-Head and Neck Surgery Foundation. Otolaryngol Neck Surg 2012; 147: 466-471 Im Internet: http://journals.sagepub.com/doi/10.1177/0194599812442783

[97] Greywoode J, Bluman E, Spiegel J, Boon M. Readability analysis of patient information on the American Academy of OtolaryngologyHead and Neck Surgery website. Otolaryngol Head Neck Surg 2009; 141: 555-558 Im Internet: http://dx.doi.org/10.1016/j. otohns.2009.08.004

[98] Atcherson SR, DeLaune AE, Hadden K, Zraick RI, Kelly-Campbell R], Minaya CP. A Computer-Based Readability Analysis of Consumer Materials on the American Speech-Language-Hearing Association Website. Contemp Issues Commun Sci Disord 2014; 41: 12-23 Im Internet http://search.ebscohost.com/login.aspx?direct = true \&db = rzh\&AN $=2012595342 \&$ site $=$ ehost - live

[99] Nassif S], Wong K, Levi JR. The Índice Flesch-Szigriszt and Spanish Lexile Analyzer to evaluate Spanish patient education materials in otolaryngology. Laryngoscope 2018; 128: E21-E26 Im Internet: http://doi.wiley.com/10.1002/lary.26910 
[100] Kasabwala K, Misra P, Hansberry DR, Agarwal N, Baredes S, Setzen M, Anderson Eloy J. Readability assessment of the American Rhinologic Society patient education materials. Int Forum Allergy Rhinol 2013; 3: 325-333 Im Internet: http://doi.wiley.com/10.1002/alr.21097

[101] Wong K, Levi JR. Readability of pediatric otolaryngology information by children's hospitals and academic institutions. Laryngoscope 2017; 127: E138-E144

[102] Hansberry DR, Agarwal N, Shah R, Schmitt P], Baredes S, Setzen M, Carmel PW, Prestigiacomo CJ, Liu JK, Eloy JA. Analysis of the readability of patient education materials from surgical subspecialties. Laryngoscope 2014; 124: 405-412 Im Internet: http://doi.wiley. com/10.1002/lary.24261

[103] Narwani V, Nalamada K, Lee M, Kothari P, Lakhani R. Readability and quality assessment of internet-based patient education materials related to laryngeal cancer. Head Neck 2016; 38: 601-605 Im Internet: http://doi.wiley.com/10.1002/hed.23939

[104] Varela-Centelles P, Ledesma-Ludi Y, Seoane-Romero JM, Seoane J. Information about oral cancer on the Internet: Our patient scannot understand it. Br J Oral Maxillofac Surg 2015; 53: 393-395 Im Internet: http://dx.doi.org/10.1016/j.bjoms.2015.01.020

[105] Irwin JY, Thyvalikakath T, Spallek H, Wali T, Kerr AR, Schleyer T. English and Spanish oral cancer information on the internet: A pilot surface quality and content evaluation of oral cancer Web sites. J Public Health Dent 2011; 71: 106-116 Im Internet http://www.ncbi. nlm.nih.gov/pubmed/21774133

[106] Wong K, Gilad A, Cohen MB, Kirke DN, Jalisi SM. Patient education materials assessment tool for laryngectomy health information. Head Neck 2017; 1-8 Im Internet http://doi.wiley.com/10.1002/ hed. 24891

[107] Albert Kong K, Hu A. Readability Assessment of Online Tracheostomy Care Resources. Otolaryngol Neck Surg 2015; 152: 272-278 Im Internet: http://journals.sagepub.com/ doi/10.1177/0194599814560338

[108] Wiriyakijja P, Fedele S, Porter S, Ni Riordain R. Web-based information on the treatment of oral leukoplakia - quality and readability. J Oral Pathol Med 2016; 45: 617-620 Im Internet: http://doi.wiley. com/10.1111/jop. 12459

[109] Ritchie L, Tornari C, Patel PM, Lakhani R. Glue ear: How good is the information on the World Wide Web? J Laryngol Otol 2016; 130: 157-161

[110] Pothier DD. Patients and the internet: are websites on glue ear readable? Clin Otolaryngol 2005; 30: 566 Im Internet: http://doi. wiley.com/10.1111/j.1749-4486.2005.01115.x

[111] Danino J, Muzaffar J, Mitchell-Innes A, Howard J, Coulson C. Quality of Information Available Via the Internet for Patients with Otological Conditions. Otol Neurotol 2016; 37: 1063-1065

[112] Joseph J, Svider PF, Shaigany K, Eloy JA, McDonald PG, Folbe AJ, Hong RS. Hearing Aid Patient Education Materials: Is There Room for Improvement? J Am Acad Audiol 2016; 27: 354-359 Im Internet http://openurl.ingenta.com $/$ content $/$ xref? genre $=$ article\&issn $=$ $1050-0545$ \& volume $=27 \&$ issue $=4 \&$ spage $=354$

[113] Seymour N, Lakhani R, Hartley B, Cochrane L, Jephson C. Cochlear implantation: An assessment of quality and readability of web-based information aimed at patients. Cochlear Implants Int 2015; 16: 321-325 Im Internet: http://www.tandfonline.com/doi/full/10.1179/ 1754762815Y.0000000015

[114] McKearney TC, McKearney RM. The quality and accuracy of internet information on the subject of ear tubes. Int J Pediatr Otorhinolaryngol 2013; 77: 894-897 Im Internet: http://dx.doi.org/10.1016/j. ijporl.2013.03.021

[115] Harris VC, Links AR, Hong P, Walsh J, Schoo DP, Tunkel DE, Stewart CM, Boss EF. Consulting Dr. Google: Quality of Online Resources About Tympanostomy Tube Placement. Laryngoscope 2018; 128: 496-501 Im Internet: http://doi.wiley.com/10.1002/lary.26824
[116] Cherla DV, Sanghvi S, Choudhry O], Jyung RW, Eloy JA, Liu JK. Readability Assessment of Internet-Based Patient Education Materials Related to Acoustic Neuromas. Otol Neurotol 2013; 34: 1349-1354 Im Internet http://content.wkhealth.com/linkback/openurl?sid= WKPTLP:landingpage\&an $=00129492-201309000-00029$

[117] Misra P, Kasabwala K, Agarwal N, Eloy JA, Liu JK. Readability analysis of internet-based patient information regarding skull base tumors. J Neurooncol 2012; 109: 573-580

[118] Haymes AT. The Quality of Rhinoplasty Health Information on the Internet. Ann Plast Surg 2016; 76: 143-149

[119] Rayess H, Gupta A, Nissan M, Carron M, Zuliani G. Search Engine Optimization: An Analysis of Rhinoplasty Web sites. Facial Plast Surg 2017; 33: 665-669 Im Internet: http://www.thieme-connect.de/DOI/ DOI?10.1055/s-0037-1607973

[120] Rayess H, Zuliani GF, Gupta A, Svider PF, Folbe AJ, Eloy JA, Carron MA. Critical analysis of the quality, readability, and technical aspects of online information provided for neck-lifts. JAMA Facial Plast Surg 2017; 19: 115-120

[121] Nissan ME, Gupta A, Rayess H, Black KZ, Carron M. Otoplasty Online Information: A Comprehensive Analysis of the Websites and Videos that Patients View Regarding Cosmetic Ear Surgery. Facial Plast Surg 2018; 34: 82-87

[122] Alamoudi U, Hong P. Readability and quality assessment of websites related to microtia and aural atresia. Int J Pediatr Otorhinolaryngol 2015; 79: 151-156 Im Internet: http://dx.doi.org/10.1016/j. ijporl.2014.11.027

[123] Acar B, Acar M, Ocak E, Kocaöz D, Koksal AO, Karasen RM. Accuracy of Internet guidance on pediatric otolaryngology procedures. Int J Pediatr Otorhinolaryngol 2014; 78: 2190-2192

[124] McGoldrick DM, Kielty P, Cotter C. Quality of information about maxillofacial trauma on the Internet. Br J Oral Maxillofac Surg 2017; 55: 141-144 Im Internet: http://dx.doi.org/10.1016/j. bjoms.2016.09.020

[125] Veer V, Alder G, Ullal U. The quality of snoring treatment information on the internet. Eur Arch Oto-Rhino-Laryngology 2014; 271: 3319-3323

[126] San Giorgi MRM, de Groot OSD, Dikkers FG. Quality and readability assessment of websites related to recurrent respiratory papillomatosis. Laryngoscope 2017; 127: 2293-2297

[127] Wong K, Levi JR. Partial Tonsillectomy: Content and Readability of Online Health Information. Ann Otol Rhinol Laryngol 2017; 126: 192-198

[128] López-Jornet P, Camacho-Alonso F. The quality of patient-orientated Internet information on oral lichen planus: A pilot study. J Eval Clin Pract 2010; 16: 883-886

[129] Kulasegarah J, Harney M, Walsh M, Walsh RM. The quality of information on three common ENT procedures on the Internet. Ir ] Med Sci 2012; 181: 221-224 Im Internet: http://link.springer. $\mathrm{com} / 10.1007 / \mathrm{s} 11845-011-0787-0$

[130] Muthukumarasamy S, Osmani Z, Sharpe A, England RJA. Quality of information available on the World Wide Web for patients undergoing thyroidectomy: Review. J Laryngol Otol 2012; 126: 116-119

[131] Ting K, Hu A. Evaluating the quality and readability of thyroplasty information on the internet. J Voice 2014; 28: 378-381 Im Internet: http://dx.doi.org/10.1016/j.jvoice.2013.10.011

[132] Cherla DV, Sanghvi S, Choudhry OJ, Liu JK, Eloy JA. Readability assessment of internet-based patient education materials related to endoscopic sinus surgery. Laryngoscope 2012; 122: 1649-1654

[133] Dueppen AJ, Bellon-Harn ML, Radhakrishnan N, Manchaiah V. Quality and Readability of English-Language Internet Information for Voice Disorders. J Voice 2017; 1-9 Im Internet: https://doi.org/10.1016/j. jvoice.2017.11.002 
[134] Alnafea S, Fedele S, Porter S, Ni Riordain R. Online Information on the Treatment of Burning Mouth Syndrome: Quality and Readability. J Oral Facial Pain Headache 2017; 31: 147-151 Im Internet: http:// www.quintpub.com/journals/ofph/abstract.php?iss2_id=1445\&article_id $=17285 \&$ article $=6 \&$ title $=$ Online Information on the Treatment of Burning Mouth Syndrome: Quality and Readability\#.WQOaDf1JmG8

[135] O'Connell Ferster AP, Hu A. Evaluating the quality and readability of Internet information sources regarding the treatment of swallowing disorders. Ear Nose Throat J 2017; 96: 128-138 Im Internet http:// www.ncbi.nlm.nih.gov/pubmed/28346643

[136] Davis KS, McCormick AA, Jabbour N. What might parents read: Sorting webs of online information on vascular anomalies. Int ] Pediatr Otorhinolaryngol 2017; 93: 63-67 Im Internet: http://dx.doi. org/10.1016/j.ijporl.2016.12.004

[137] Sanghvi S, Cherla DV, Shukla PA, Eloy JA. Readability assessment of internet-based patient education materials related to facial fractures. Laryngoscope 2012; 122: 1943-1948

[138] Laplante-Lévesque A, Brännström KJ, Andersson G, Lunner T. Quality and readability of English-language internet information for adults with hearing impairment and their significant others. Int J Audiol 2012; 51: 618-626

[139] Goslin RA, Elhassan HA. Evaluating internet health resources in ear, nose, and throat surgery. Laryngoscope 2013; 123: 1626-1631 Im Internet: http://doi.wiley.com/10.1002/lary.23773

[140] Laplante-Lévesque A, Thorén ES. Readability of Internet Information on Hearing: Systematic Literature Review. Am J Audiol 2015; 24: 284 Im Internet: http://aja.pubs.asha.org/article. aspx?doi $=10.1044 / 2015 \_$AJA-14-0091

[141] Hardey M. Public health and Web 2.0. J R Soc Promot Health 2008; 128: 181-189 Im Internet: http://rsh.sagepub.com/cgi/ doi/10.1177/1466424008092228

[142] Hesse BW, O'Connell M, Augustson EM, Chou W-YS, Shaikh AR, Rutten LJF. Realizing the promise of Web 2.0: engaging community intelligence. J Health Commun 2011; 16: (Suppl 1): 10-31 Im Internet: http://www.tandfonline.com/doi/abs/10.1080/10810730.2 011.589882

[143] Lau AYS, Siek KA, Fernandez-Luque L, Tange H, Chhanabhai P, Li SYW, Elkin PL, Arjabi A, Walczowski L, Ang CS, Eysenbach G. The Role of Social Media for Patients and Consumer Health. Yearb Med Inform 2011; 20: 131-138 Im Internet: http://www.thieme-connect.de/DOI/ DOI?10.1055/s-0038-1638751

[144] Feng Y, Xie W. Digital divide 2.0: the role of social networking sites in seeking health information online from a longitudinal perspective. J Health Commun 2015; 20: 60-68 Im Internet: http://www. tandfonline.com/doi/abs/10.1080/10810730.2014.906522

[145] Steele SR, Arshad S, Bush R, Dasani S, Cologne K, Bleier JIS, Raphaeli $\mathrm{T}$, Kelz RR. Social media is a necessary component of surgery practice. Surg (United States) 2015; 158: 857-862 Im Internet: http://dx.doi.org/10.1016/j.surg.2015.06.002

[146] Steehler KR, Steehler MK, Pierce ML, Harley EH. Social media's role in otolaryngology-head and neck surgery: Informing clinicians, empowering patients. Otolaryngol Head Neck Surg 2013; 149: 521-524 Im Internet: http://journals.sagepub.com/ doi/10.1177/0194599813501463

[147] Farnan JM, Snyder Sulmasy L, Worster BK, Chaudhry HJ, Rhyne JA, Arora VM.American College of Physicians Ethics P and HRC, American College of Physicians Council of Associates, Federation of State Medical Boards Special Committee on Ethics and Professionalism*. Online medical professionalism: patient and public relationships: policy statement from the American College of Physicians and the Federation of State Medical Boards. Ann Intern Med 2013; 158: 620-627 Im Internet: http://annals.org/article.aspx?doi = 10 . 7326/0003-4819-158-8-201304160-00100
[148] Walsh K. Social media and surgery: An alternative view. Surg (United States) 2016; 159: 978 Im Internet: http://dx.doi.org/10.1016/j. surg.2015.07.032

[149] Park H, Rodgers S, Stemmle J. Analyzing health organizations' use of twitter for promoting health literacy. J Health Commun 2013; 18: 410-425

[150] Pershad Y, Hangge P, Albadawi H, Oklu R. Social Medicine: Twitter in Healthcare. J Clin Med 2018; 7: 121 Im Internet http://www.mdpi. com/2077-0383/7/6/121

[151] Shafee T, Masukume G, Kipersztok L, Das D, Häggström M, Heilman J. Evolution of Wikipedia's medical content: past, present and future. J Epidemiol Community Health 2017; 71: 1122-1129 Im Internet: http://jech.bmj.com/lookup/doi/10.1136/jech-2016-208601

[152] Heilman JM, Kemmann E, Bonert M, Chatterjee A, Ragar B, Beards GM, Iberri DJ, Harvey M, Thomas B, Stomp W, Martone MF, Lodge DJ, Vondracek A, de Wolff JF, Liber C, Grover SC, Vickers TJ, Meskó B, Laurent MR. Wikipedia: a key tool for global public health promotion. J Med Internet Res 2011; 13: e14 Im Internet: http://www.jmir. org/2011/1/e14/

[153] Laurent MR, Vickers TJ. Seeking health information online: does Wikipedia matter? J Am Med Inform Assoc 2009; 16: 471-479 Im Internet: http://dx.doi.org/10.1197/jamia.M3059

[154] Heilman JM, West AG. Wikipedia and Medicine: Quantifying Readership, Editors, and the Significance of Natural Language. J Med Internet Res 2015; 17: e62 Im Internet: http://www.jmir.org/2015/3/e62/

[155] Zhang B. YouTube for Patient Education : A Deep Learning Approach for Understanding Medical Knowledge from User-Generated Videos. 2018; 2-4

[156] Madathil KC, Rivera-Rodriguez A], Greenstein JS, Gramopadhye AK. Healthcare information on YouTube: A systematic review. Health Informatics J 2015; 21: 173-194 Im Internet: http://journals. sagepub.com/doi/10.1177/1460458213512220

[157] Stamelou M, Edwards M], Espay A], Fung VSC, Hallett M, Lang AE, Tijssen MAJ, Bhatia KP. Movement Disorders on YouTube - Caveat Spectator. N Engl J Med 2011; 365: 1160-1161 Im Internet: http:// www.nejm.org/doi/abs/10.1056/NEJMc1107673

[158] Knight K, van Leeuwen DM, Roland D, Moll HA, Oostenbrink R. YouTube: Are parent-uploaded videos of their unwell children a useful source of medical information for other parents? Arch Dis Child 2017; 102: 910-914 Im Internet: http://adc.bmj.com/lookup/ doi/10.1136/archdischild-2016-311967

[159] Fat MJL, Doja A, Barrowman N, Sell E. YouTube videos as a teaching tool and patient resource for infantile spasms. J Child Neurol 2011; 26: 804-809 Im Internet: http://journals.sagepub.com/ doi/10.1177/0883073811402345

[160] Delli K, Livas C, Vissink A, Spijkervet FKLL. Is YouTube useful as a source of information for Sjögren's syndrome? Oral Dis 2016; 22: 196-201 Im Internet: http://doi.wiley.com/10.1111/odi.12404

[161] Nissan ME, Gupta A, Carron J, Rayess H, Carron M. Rhytidectomy: Analysis of Videos Available Online. Facial Plast Surg 2017; 33: 311-315 Im Internet: http://www.thieme-connect.de/DOI/ DOI?10.1055/s-0037-1602163

[162] Sorensen JA, Pusz MD, Brietzke SE. YouTube as an information source for pediatric adenotonsillectomy and ear tube surgery. Int J Pediatr Otorhinolaryngol 2014; 78: 65-70 Im Internet http://linkinghub. elsevier.com/retrieve/pii/S0165587613005314

[163] Hassona Y, Taimeh D, Marahleh A, Scully C. YouTube as a source of information on mouth (oral) cancer. Oral Dis 2016; 22: 202-208 Im Internet http://doi.wiley.com/10.1111/odi.12434

[164] Biggs TC, Bird JH, Harries PG, Salib RJ. YouTube as a source of information on rhinosinusitis: the good, the bad and the ugly. J Laryngol Otol 2013; 127: 749-754 Im Internet http://www.journals. cambridge.org/abstract_S0022215113001473 
[165] Eysenbach G, Powell ], Englesakis M, Rizo C, Stern A. Health related virtual communities and electronic support groups: systematic review of the effects of online peer to peer interactions. BMJ 2004; 328: 1166 Im Internet http://www.ncbi.nlm.nih.gov/pubmed/15142921\%5Cnhttp://www.pubmedcentral.nih.gov/articlerender.fcgi?artid = PMC411092

[166] Brownstein CA, Brownstein JS, Williams DS, Wicks P, Heywood JA. The power of social networking in medicine. Nat Biotechnol 2009; 27: 888-890 Im Internet http://www.nature.com/articles/nbt1009-888

[167] Fornai F, Longone P, Cafaro L, Kastsiuchenka O, Ferrucci M, Manca ML, Lazzeri G, Spalloni A, Bellio N, Lenzi P, Modugno N, Siciliano G, Isidoro C, Murri L, Ruggieri S, Paparelli A. Lithium delays progression of amyotrophic lateral sclerosis. Proc Natl Acad Sci U S A 2008; 105: 2052-2057 Im Internet http://www.ncbi.nlm.nih.gov/pubmed/18250315

[168] Wicks P, Vaughan TE, Massagli MP, Heywood J. Accelerated clinical discovery using self-reported patient data collected online and a patient-matching algorithm. Nat Biotechnol 2011; 29: 411-414 Im Internet http://www.nature.com/articles/nbt.1837

[169] Frost J, Okun S, Vaughan T, Heywood J, Wicks P. Patient-reported outcomes as a source of evidence in off-label prescribing: analysis of data from PatientsLikeMe. J Med Internet Res 2011; 13: e6 Im Internet http://www.jmir.org/2011/1/e6/

[170] Reifegerste D, Baumann E. Medien und Gesundheit..Wiesbaden: Springer Fachmedien Wiesbaden; 2018Im Internet http://link. springer.com/10.1007/978-3-658-20013-8

[171] Ad Hoc Committee on Health Literacy for the Council on Scientific Affairs American Medical Association. Health Literacy: Report of the Council on Scientific Affairs. JAMA J Am Med Assoc 1999; 281: 552-557 Im Internet http://www.ncbi.nlm.nih.gov/pub$\operatorname{med} / 22442354$

[172] Sørensen K, Van den Broucke S, Fullam J, Doyle G, Pelikan J, Slonska $\mathrm{Z}$, Brand $\mathrm{H}$. Health literacy and public health: A systematic review and integration of definitions and models. BMC Public Health 2012; 12: 80 Im Internet http://bmcpublichealth.biomedcentral.com/ articles/10.1186/1471-2458-12-80

[173] Levin-Zamir D, Bertschi I. Media Health Literacy, eHealth Literacy, and the Role of the Social Environment in Context. Int J Environ Res Public Health 2018; 15: 1643 Im Internet http://www.mdpi. com/1660-4601/15/8/1643

[174] Norman CD, Skinner HA. eHealth Literacy: Essential Skills for Consumer Health in a Networked World. J Med Internet Res 2006; 8: e9 Im Internet http://www.jmir.org/2006/2/e9/

[175] Griebel L, Enwald H, Gilstad H, Pohl A-L, Moreland J, Sedlmayr M. eHealth literacy research-Quo vadis? Informatics Heal Soc Care 2017; 00: 1-16 Im Internet https://doi.org/10.1080/17538157.2017. 1364247

[176] DeWalt DA, Berkman ND, Sheridan S, Lohr KN, Pignone MP. Literacy and health outcomes. J Gen Intern Med 2004; 19: 1228-1239 Im Internet http://link.springer.com/10.1111/j.1525-1497.2004.40153.x

[177] Berkman ND, Sheridan SL, Donahue KE, Halpern DJ, Crotty K. Low Health Literacy and Health Outcomes: An Updated Systematic Review. Ann Intern Med 2011; 155: 97 Im Internet http://annals.org/ article. aspx?doi $=10.7326 / 0003-4819-155-2-201107190-00005$

[178] Bostock S, Steptoe A. Association between low functional health literacy and mortality in older adults: longitudinal cohort study. BM] 2012; 344: e1602 Im Internet http://www.ncbi.nlm.nih.gov/ pubmed/22422872

[179] Eysenbach G. The Impact of the Internet on Cancer Outcomes. CA Cancer J Clin 2003; 53: 356-371 Im Internet http://doi.wiley. com/10.3322/canjclin.53.6.356
[180] Diviani N, van den Putte B, Giani S, van Weert JCMC. Low health literacy and evaluation of online health information: A systematic review of the literature. J Med Internet Res 2015; 17: 1-17 Im Internet http://www.ncbi.nlm.nih.gov/pubmed/25953147

[181] Zach L, Dalrymple PW, Rogers ML, Williver-Farr H. Assessing Internet access and use in a medically underserved population: Implications for providing enhanced health information services. Health Info Libr J 2012; 29: 61-71

[182] Ramanadhan S, Viswanath K. Health and the Information Nonseeker: A Profile. Health Commun 2006; 20: 131-139 Im Internet http:// www.tandfonline.com/doi/abs/10.1207/s15327027hc2002_4

[183] Megwalu UC, Lee JY. Health Literacy Assessment in an Otolaryngology Clinic Population. Otolaryngol Neck Surg 2016; 155: 969-973 Im Internet http://journals.sagepub.com/ doi/10.1177/0194599816664331

[184] Safeer RS, Keenan J. Health literacy: The gap between physicians and patients. Am Fam Physician 2005; 72: 463-468 Im Internet https:// www.aafp.org/afp/2005/0801/p463.html

[185] Schaeffer D, Berens E-M, Vogt D. Gesundheitskompetenz der Bevölkerung in Deutschland: Ergebnisse einer repräsentativen Befragung. Dtsch Aerzteblatt Online. 2017; 114 Im Internet https:// www.aerzteblatt.de/10.3238/arztebl.2017.0053

[186] Jordan S, Hoebel J. Gesundheitskompetenz von Erwachsenen in Deutschland. Bundesgesundheitsblatt - Gesundheitsforsch - Gesundheitsschutz 2015; 58: 942-950 Im Internet http://link.springer. com/10.1007/s00103-015-2200-z

[187] Sørensen K, Pelikan JM, Röthlin F, Ganahl K, Slonska Z, Doyle G, Fullam J, Kondilis B, Agrafiotis D, Uiters E, Falcon M, Mensing M, Tchamov K, van den Broucke S, Brand H. HLS-EU Consortium. Health literacy in Europe: comparative results of the European health literacy survey (HLS-EU). Eur J Public Health 2015; 25: 1053-1058 Im Internet https://academic.oup.com/eurpub/article-lookup/ doi/10.1093/eurpub/ckv043

[188] Nölke L, Mensing M, Krämer A, Hornberg C. Sociodemographic and health-(care-)related characteristics of online health information seekers: a cross-sectional German study. BMC Public Health 2015; 15: 31 Im Internet http://bmcpublichealth.biomedcentral.com/ articles/10.1186/s12889-015-1423-0

[189] Kruse RL, Koopman RJ, Wakefield BJ, Wakefield DS, Keplinger LE, Canfield SM, Mehr DR. Internet use by primary care patients: where is the digital divide? Fam Med 2012; 44: 342-347 Im Internet http:// www.ncbi.nlm.nih.gov/pubmed/23027117

[190] Zok K. Unterschiede bei der Gesundheitskompetenz. Ergebnisse einer bundesweiten Repräsentativ-Umfrage unter gesetzlich Versicherten. WIdO-monitor 2014; 11: 1-12

[191] Rogers SN, Rozek A, Aleyaasin N, Promod P, Lowe D. Internet use among head and neck cancer survivors in the North West of England. Br J Oral Maxillofac Surg 2012; 50: 208-214 Im Internet http://dx.doi. org/10.1016/j.bjoms.2011.03.264

[192] Sheridan SL, Halpern D], Viera AJ, Berkman ND, Donahue KE, Crotty K. Interventions for individuals with low health literacy: A systematic review. J Health Commun 2011; 16: 30-54

[193] Beerheide R. Ministerium sucht Schulterschluss. Dtsch Arztebl 2017; 114: A1223

[194] Tan SS-L, Goonawardene N. Internet Health Information Seeking and the Patient-Physician Relationship: A Systematic Review. J Med Internet Res 2017; 19: e9 Im Internet http://www.jmir.org/2017/1/e9/

[195] Ball M], Lillis J. E-health: transforming the physician/patient relationship. Int J Med Inform 2001; 61: 1-10 Im Internet http:// linkinghub.elsevier.com/retrieve/pii/S1386505600001301

[196] Jutel A. “Dr. Google” and his predecessors. Diagnosis 2017; 0: 87-91 Im Internet http://www.degruyter.com/view/j/dx.ahead-of-print/ dx-2016-0045/dx-2016-0045.xml 
[197] Hartzband P, Groopman J. Untangling the Web - Patients, Doctors, and the Internet. N Engl J Med 2010; 362: 1063-1066 Im Internet http://www.nejm.org/doi/abs/10.1056/NEJMp0911938

[198] Murray E, Lo B, Pollack L, Donelan K, Catania J, Lee K, Zapert K, Turner $R$. The impact of health information on the Internet on health care and the physician-patient relationship: National U.S. survey among 1.050 U.S. Physicians. J Med Internet Res 2003; 5: 38-53

[199] Davis JK. Dr. Google and Premature Consent: Patients Who Trust the Internet More Than They Trust Their Provider. HEC Forum 2017; 1-13

[200] Woolf SH, Chan ECY, Harris R, Sheridan SL, Braddock CH, Kaplan RM, Krist A, O'Connor AM, Tunis S. Promoting Informed Choice: Transforming Health Care To Dispense Knowledge for Decision Making. Ann Intern Med 2005; 143: 293 Im Internet http://annals. org/article. aspx?doi = 10.7326/0003-4819-143-4-200508160-00010

[201] Sommerhalder K, Abraham A, Zufferey MC, Barth J, Abel T. Internet information and medical consultations: Experiences from patients' and physicians' perspectives. Patient Educ Couns 2009; 77: 266-271

[202] Hart A, Henwood F, Wyatt S. The role of the internet in patient-practitioner relationships: Findings from a qualitative research study. J Med Internet Res 2004; 6: 1-7

[203] Fergus TA, Dolan SL. Problematic internet use and internet searches for medical information: the role of health anxiety. Cyberpsychol Behav Soc Netw 2014; 17: 761-765 Im Internet http://online. liebertpub.com/doi/abs/10.1089/cyber.2014.0169

[204] Ellingson MK, Bonk CM, Chamberlain AT. A survey-based study of Zika virus communication preferences among pregnant women in Georgia, United States. BMC Pregnancy Childbirth 2017; 17: 325 Im Internet http://bmcpregnancychildbirth.biomedcentral.com/ articles/10.1186/s12884-017-1516-0
[205] Hesse BW, Moser RP, Rutten LJ. Surveys of Physicians and Electronic Health Information. N Engl J Med 2010; 362: 859-860 Im Internet http://www.nejm.org/doi/abs/10.1056/NEJMc0909595

[206] Illingworth P. Trust: The Scarcest of Medical Resources. J Med Philos 2002; 27: 31-46 Im Internet https://academic.oup.com/jmp/ article-lookup/doi/10.1076/jmep.27.1.31.2969

[207] Ahmad F, Hudak PL, Bercovitz K, Hollenberg E, Levinson W. Are Physicians Ready for Patients With Internet-Based Health Information? J Med Internet Res 2006; 8: e22 Im Internet http://www.jmir. org/2006/3/e22/

[208] Wayne I. Ethical considerations in revision rhinoplasty. Facial Plast Surg 2012; 28: 369-373

[209] Szychta P, Zieliński T, Rykała J, Witmanowski H, Kruk-Jeromin J. Role of the internet in communication between patient and surgeon before rhinoplasty. J Plast Surg Hand Surg 2012; 46: 248-251

[210] Balakrishnan V, Chandy Z, Verma SP. Are Online Zenker's Diverticulum Materials Readable and Understandable? Otolaryngol - Head Neck Surg (United States) 2016; 155: 758-763

[211] Xie DX, Wang RY, Chinnadurai S. Readability of online patient education materials for velopharyngeal insufficiency. Int J Pediatr Otorhinolaryngol 2018; 104: 113-119 Im Internet https://doi. org/10.1016/j.ijporl.2017.09.016

[212] Wozney L, Chorney J, Huguet A, Song JS, Boss EF, Hong P. Online Tonsillectomy Resources: Are Parents Getting Consistent and Readable Recommendations? Otolaryngol - Head Neck Surg (United States) 2017 ; 156: 844-852 